\title{
SYSTEMS OF DIAGRAM CATEGORIES AND $K$-THEORY. I
}

\author{
G. GARKUSHA
}

\begin{abstract}
With any left system of diagram categories or any left pointed dérivateur, a $K$-theory space is associated. This $K$-theory space is shown to be canonically an infinite loop space and to have a lot of common properties with Waldhausen's $K$ theory. A weaker version of additivity is shown. Also, Quillen's $K$-theory of a large class of exact categories including the Abelian categories is proved to be a retract of the $K$-theory of the associated dérivateur.
\end{abstract}

\section{INTRODUCTION}

The notion of a dérivateur was introduced by Grothendieck in [1, 2]. Independently, similar constructions were investigated by Heller [3], Keller [4], and Franke [5] (the so-called system of diagram categories). Basing on papers by Grothendieck [2] and Franke [5], Maltsiniotis [6] introduced the notion of a triangulated dérivateur $\mathbf{D}$, and then (together with Keller) constructed the $K$-theory space $K(\mathbf{D})$ for $\mathbf{D}$. In his paper [7] on the $K$-theory for triangulated dérivateurs, Maltsiniotis suggested three conjectures. Our goal in this paper is to give partial affirmative solutions to the additivity and comparison conjectures.

It should be mentioned that we study not only triangulated dérivateurs but also the $K$-theory $K(\mathbf{B})$ of more general objects $\mathbf{B}$ as, for example, left systems of diagram categories or left pointed dérivateurs. There are plenty of such objects in practice.

First we define the $S$.-construction for such B's. Then the $K$-theory space $K(\mathbf{B})$ is defined as the loop space for $|i . S . \mathbf{B}|$, where $i S_{n} \mathbf{B}$ is the subcategory of isomorphisms in each category $S_{n} \mathbf{B}, n \geq 0$. The space $K(\mathbf{B})$ is canonically an infinite loop space by Segal's machine [8]. As in Waldhausen's $K$-theory [9], the additivity theorem implies that the space $K(\mathbf{B})$ can be considered in terms of the following connected $\Omega$-spectrum. Precisely, it is given by the sequence of spaces

$$
\Omega|i . S . \mathbf{B}|, \quad \Omega|i . S . S . \mathbf{B}|, \ldots, \quad \Omega\left|i . S .{ }^{n} \mathbf{B}\right|, \ldots,
$$

where the multisimplicial objects $i . S .{ }^{n} \mathbf{B}, n \geq 1$, are obtained by iterating the $S$.-construction. Though the additivity theorem remains open in the general case (see also [7, Conjecture 3]), a weaker version holds true.

Theorem 6.5. The additivity theorem holds for the space $\Omega^{\infty}\left|i . S .{ }^{\infty} \mathbf{B}\right|=\lim _{n} \Omega^{n}\left|i . S .{ }^{n} \mathbf{B}\right|$.

In fact, this theorem is proved under certain additional natural assumptions (see below).

The strong form of additivity was proved for complicial dérivateurs in [10]. Very recently, Cisinski and Neeman proved that additivity is true for triangulated dérivateurs [11].

2000 Mathematics Subject Classification. Primary 19 D99.

Key words and phrases. Systems of diagram categories, Grothendieck's dérivateurs, algebraic $K$ theory.

Supported by the ICTP Research Fellowship. 
We denote by $\mathbf{D}^{b}(\mathcal{E})$ the dérivateur which is defined as the hypofunctor $I \mapsto D^{b}\left(\mathcal{E}^{I}\right)$, where $D^{b}\left(\mathcal{E}^{I}\right)$ is the derived category for the exact functor category $\mathcal{E}^{I}$. Some relations between Quillen's $K$-theory $K(\mathcal{E})$ and the $K$-theory $K\left(\mathbf{D}^{b}(\mathcal{E})\right)$ can be obtained for a large class of exact categories including the Abelian categories.

Theorem 7.1. Let $\mathcal{E}$ be an extension closed, full, exact subcategory of an Abelian category $\mathcal{A}$ satisfying the conditions of the resolution theorem. That is,

(1) if $0 \longrightarrow M^{\prime} \longrightarrow M \longrightarrow M^{\prime \prime} \longrightarrow 0$ is exact in $\mathcal{A}$ and $M, M^{\prime \prime} \in \mathcal{E}$, then $M^{\prime} \in \mathcal{E}$;

(2) for any object $M \in \mathcal{A}$ there is a finite resolution $0 \longrightarrow P_{n} \longrightarrow P_{n-1} \longrightarrow \cdots \longrightarrow$ $P_{0} \longrightarrow M \longrightarrow 0$ with $P_{i} \in \mathcal{E}$.

Then a natural map

$$
K(\rho): K(\mathcal{E}) \longrightarrow K\left(\mathbf{D}^{b}(\mathcal{E})\right)
$$

has the property that, for some map $p: K\left(\mathbf{D}^{b}(\mathcal{E})\right) \longrightarrow K(\mathcal{E})$, the map $p \circ K(\rho)$ is homotopic to the identity map. In particular, each $K$-group $K_{n}(\mathcal{E})$ is a direct summand of $K_{n}\left(\mathbf{D}^{b}(\mathcal{E})\right)$.

Theorem 7.1 gives a partial affirmative solution to the comparison conjecture of Maltsiniotis. It also shows that the $K$-theory of dérivateurs is of a fairly complicated nature.

I would like to thank Denis-Charles Cisinski and Amnon Neeman for helpful discussions. I am also very grateful to A. I. Generalov for a careful reading of the manuscript.

\section{§1. Systems of Diagram CATEgories}

In this section the reader will face categorical formalities and definitions of concepts. A lot of similar statements can also be found in [5]. Here we follow the original terminology of Franke [5].

1.1. Notation. Let $I$ be a category. For a subcategory $J$ of $I$ and $x \in I$, we shall denote by $J / x$ the following comma category. The objects are the pairs $(y, \varphi)$, where $y \in J$ and $\varphi: y \longrightarrow x$ is a morphism in $I$. The morphisms from $(y, \varphi)$ to $\left(y^{\prime}, \varphi^{\prime}\right)$ are given by morphisms $\psi: y \longrightarrow y^{\prime}$ in $J$ such that $\varphi=\varphi^{\prime} \psi$. The category $J \backslash x$ consists of pairs $(y, \varphi)$ with $y \in J$ and $\varphi: x \longrightarrow y$. The morphisms are similar to those of $J / x$. If $K \subseteq \mathrm{Ob} I$ is a subclass of objects, we denote by $I-K$ the full subcategory of $I$ with the class of objects $I-K$. In particular, if $K=\{x\}$ has only one object, we still denote this subcategory by $I-x$. If $f: J \longrightarrow I$ is a functor, the categories $f / x$ and $f \backslash x$ have objects $(y \in J, \varphi: f(y) \longrightarrow x)$ and $(y \in J, \varphi: x \longrightarrow f(y))$. If $f$ is the inclusion of a subcategory, these categories coincide with $J / x$ and $J \backslash x$.

Given a nonnegative integer $n$, we denote by $\Delta^{n}$ the totally ordered set $\{0<1<\cdots<$ $n\}$. For $i \leq n+1$, the map $d_{i}: \Delta^{n} \longrightarrow \Delta^{n+1}$ is the monotone injection not containing $i$ in its image, and $s_{i}: \Delta^{n} \longrightarrow \Delta^{n-1}$ is the monotone surjection satisfying $s_{i}(i)=s_{i}(i+1)$.

1.2. Axioms. For the notions of the 2 -category and the 2 -functor, we refer the reader to [12]. In what follows we use the term "poset" as an abbreviation of "finite partially ordered set". Every poset can be regarded as a category in which $\operatorname{Hom}(x, y)$ has precisely one element if $x \leq y$, and is empty otherwise. The 2-category of posets (respectively, of finite categories without cycles) will be denoted by Ord (respectively by Dirf).

Let $\mathcal{D}$ ia be a full 2-subcategory of the 2-category Cat of small categories that contains the 2-category Ord. In what follows we assume that $\mathcal{D}$ ia satisfies the following conditions:

(1) Dia is closed under finite sums and finite products;

(2) for any functor $f: I \longrightarrow J$ in $\mathcal{D}$ ia and for any object $y$ of $J$, the categories $f / y$ and $f \backslash y$ are in $\mathcal{D}$ ia. 
We shall also refer to $\mathcal{D}$ ia as a category of diagrams.

Given $I \in \mathcal{D}$ ia, let $I^{\star}$ be the category $I$ with an initial and final object $\star$ added. For any $x$ and $y$ in $I$, there is a unique morphism from $x$ to $y$ in $I^{\star}$ that factorizes through $\star$. We shall refer to this morphism as the zero morphism. If $I \in$ Ord and $x \leq y$, there is yet another morphism from $x$ to $y$ in $I^{\star}$. Composition is defined in an obvious way. Let $\mathcal{D i a}^{\star}$ be the 2 -subcategory of the 2 -category Cat. Its objects are those of $\mathcal{D}$ ia, and its horizontal morphisms $I \longrightarrow J$ are given by functors $I^{\star} \longrightarrow J^{\star}$ mapping $\star$ to $\star$. Let bimorphisms be natural transformations between functors from $I^{\star}$ to $J^{\star}$. Note that every morphism $f: I \longrightarrow J$ in $\mathcal{D}$ ia extends naturally to a morphism $f^{\star}: I \longrightarrow J$ from $\mathcal{D i a}^{\star}$ : $f^{\star}(I)=f(I), f^{\star}(\star):=\star$.

A presystem of diagram categories with domain Dia or simply a presystem of diagram categories is a functor

$$
\mathbf{C}: \mathcal{D i a}^{\star \mathrm{op}} \longrightarrow \mathbf{C A T}
$$

from $\mathcal{D i a}^{\star}$ to the category $\mathbf{C A T}$ of categories (not necessarily small) that satisfies the functoriality axiom (see below). So, with each category $I$ in $\mathcal{D i a}^{\star}$, a category $\mathbf{C}_{I}$ is associated and each map $f: I \longrightarrow J$ in $\mathcal{D i a}^{\star}$ gives rise to a functor $f^{*}=\mathbf{C}(f): \mathbf{C}_{J} \longrightarrow$ $\mathbf{C}_{I}$.

Functoriality axiom. The following conditions are satisfied:

(a) with each natural transformation $\varphi: f \longrightarrow g$, a natural transformation $\varphi^{*}$ : $f^{*} \longrightarrow g^{*}$ is associated, and the maps $f \longrightarrow f^{*}$ and $\varphi \longrightarrow \varphi^{*}$ determine a functor from $\operatorname{Hom}(I, J)$ to the category of functors from $\mathbf{C}_{J}$ to $\mathbf{C}_{I}$;

(b) if

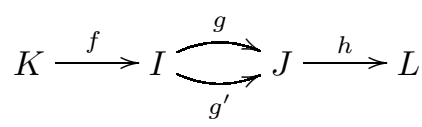

are morphisms and $\varphi: g \longrightarrow g^{\prime}$ is a bimorphism, then $f^{*} \circ \varphi^{*}=(\varphi \circ f)^{*}$ and $\varphi^{*} \circ h^{*}=$ $(h \circ \varphi)^{*}$.

From now on, we fix a category of diagrams $\mathcal{D}$ ia. With any category $\mathcal{C}$ we associate a presystem of diagram categories that takes a category $I$ in $\mathcal{D}$ ia $^{\star}$ to the functor category

$$
\mathcal{C}^{I^{\star}}=\operatorname{Hom}\left(I^{\star}, \mathcal{C}\right)
$$

and a map $f: I \longrightarrow J$ to the map

$$
f^{*}: \mathcal{C}^{J^{\star}} \longrightarrow \mathcal{C}^{I^{\star}}, \quad X \mapsto X \circ f .
$$

A morphism $F: \mathbf{C} \longrightarrow \mathbf{C}^{\prime}$ between two presystems $\mathbf{C}$ and $\mathbf{C}^{\prime}$ of diagram categories consists of the following data:

(1) for any $I \in \mathcal{D}$ ia $^{\star}$, a functor $F: \mathbf{C}_{I} \longrightarrow \mathbf{C}_{I}^{\prime}$ is given;

(2) for any map $f: I \longrightarrow J$ in $\mathcal{D i a}^{\star}$, there is an isomorphism of functors $\iota_{F, f}$ : $f^{*} F \stackrel{\sim}{\longrightarrow} F f^{*}$.

We also assume that $\iota_{F, f}$ satisfies the following conditions:

(a) $\iota_{F, 1_{I}}=1_{F}$ for any $I \in \mathcal{D}$ ia $^{\star}$;

(b) for any two maps $I \stackrel{f}{\longrightarrow} J \stackrel{g}{\longrightarrow} K$ in $\mathcal{D i a}^{\star}$, the diagram

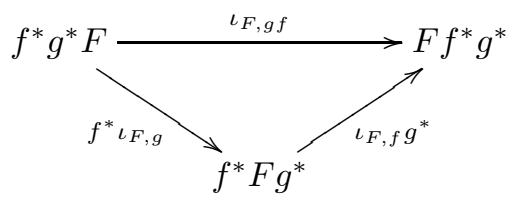

is commutative; 
(c) for any bimorphism $\varphi: f \longrightarrow g$ in $\mathcal{D}$ ia $^{\star}$, we have the following commutative square:

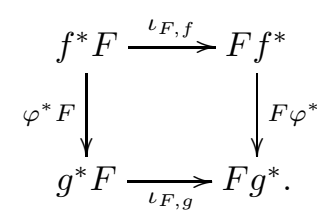

A morphism $F: \mathbf{C} \longrightarrow \mathbf{C}^{\prime}$ is an equivalence if for any $I \in \mathcal{D}$ ia ${ }^{\star}$ the functor $F: \mathbf{C}_{I} \longrightarrow \mathbf{C}_{I}^{\prime}$ is an equivalence of categories.

Let $\mathbf{A}, \mathbf{B}, \mathbf{C}$ be presystems of diagram categories. By the fiber product of a pair of morphisms $F: \mathbf{A} \longrightarrow \mathbf{C}$ and $G: \mathbf{B} \longrightarrow \mathbf{C}$ we mean the following data:

(a) for any $I \in \mathcal{D i a}^{\star}$, we have the category $\prod(F, G)_{I}$ whose objects are the triples

$$
(A, c, B), \quad A \in \mathbf{A}_{I}, \quad B \in \mathbf{B}_{I}, \quad c: F(A) \stackrel{\sim}{\longrightarrow} G(B),
$$

and where a morphism from $(A, c, B)$ to $\left(A^{\prime}, c^{\prime}, B^{\prime}\right)$ is a pair of morphisms $(a, b)$ compatible with the isomorphisms $c$ and $c^{\prime}$;

(b) for any map $f: I \longrightarrow J$ in $\mathcal{D i a}^{\star}$, we have a functor

$$
f^{*}=f_{\prod(F, G)}^{*}: \prod(F, G)_{J} \longrightarrow \prod(F, G)_{I},
$$

defined by

$$
(A, c, B) \mapsto\left(f_{\mathbf{A}}^{*}(A), \iota_{G, f} \circ f_{\mathbf{C}}^{*}(c) \circ \iota_{F, f}^{-1}, f_{\mathbf{B}}^{*}(B)\right) .
$$

Proposition 1.1. The above data determine a presystem of diagram categories

$$
\prod(F, G): \mathcal{D i a}^{\star o p} \longrightarrow \mathbf{C A T} .
$$

Proof. We show that (2) is a functor. Consider two composable maps $I \stackrel{g}{\longrightarrow} J \stackrel{f}{\longrightarrow} K$ in Dia $^{\star}$. We have

$$
\begin{aligned}
\iota_{G, g} f^{*} \circ g^{*}\left(\iota_{G, f} f^{*}(c) \iota_{F, f}^{-1}\right) \circ \iota_{F, g}^{-1} f^{*} \\
=\underbrace{\iota_{G, g} f^{*} \circ g^{*}\left(\iota_{G, f}\right)}_{\iota_{G, f g}} \circ g^{*}\left(f^{*}(c)\right) \circ \overbrace{g^{*}\left(\iota_{F, f}^{-1}\right) \circ \iota_{F, g}^{-1} f^{*}}^{\iota_{F, f g}^{-1}}=\iota_{G, f g}(f g)^{*}(c) \iota_{F, f g}^{-1} .
\end{aligned}
$$

Thus, $(f g)^{*}=g^{*} f^{*}: \prod(F, G)_{K} \longrightarrow \prod(F, G)_{I}$.

Since the diagram

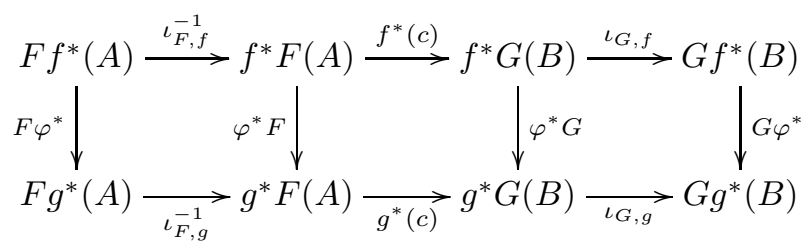

is commutative for any morphisms $f, g: I \longrightarrow J$ and any bimorphism $\varphi: f \longrightarrow g$ in $\mathcal{D i a}^{\star}$, the map

$$
\left(f^{*}(A), \iota_{G, f} f^{*}(c) \iota_{F, f}^{-1}, f^{*}(B)\right) \mapsto\left(g^{*}(A), \iota_{G, g} g^{*}(c) \iota_{F, g}^{-1}, g^{*}(B)\right)
$$

yields a map $\varphi^{*}$ between $f^{*}, g^{*}: \prod(F, G)_{J} \longrightarrow \prod(F, G)_{I}$. The functoriality axiom is verified by direct inspection. 
Let $\Delta^{n}=\{0<\cdots<n\} \in \mathcal{D}$ ia. If no confusion is possible, we also denote $\Delta^{0}$ by 0 . Given $I \in \mathcal{D}$ ia and $x \in I$, we let $i_{x, I}: 0 \longrightarrow I$ be the functor that sends 0 to $x$. For $A \in \mathbf{C}_{I}$, put $A_{x}=i_{x, I}^{*} A$.

If $I \in \mathcal{D}$ ia, there is a natural functor

$$
\operatorname{dia}_{I}: \mathbf{C}_{I} \longrightarrow \operatorname{Hom}\left(I, \mathbf{C}_{0}\right)
$$

constructed as follows. For any $x \in I$ we put $\operatorname{dia}_{I}(B)(x)=B_{x}$. Every morphism $\alpha: x \longrightarrow y$ in $I$ yields a natural transformation $\alpha: i_{x, I} \longrightarrow i_{y, I}$. Then $\operatorname{dia}_{I}(B)(\alpha):=$ $\alpha^{*}: B_{x} \longrightarrow B_{y}$.

In what follows we shall need the following axioms.

Isomorphism axiom. A morphism $f: A \longrightarrow B$ in $\mathbf{C}_{I}$ is an isomorphism if and only if $\operatorname{dia}_{I}(f)$ is an isomorphism in $\operatorname{Hom}\left(I, \mathbf{C}_{0}\right)$. In other words, it is an isomorphism if and only if $f_{x}: A_{x} \longrightarrow B_{x}$ is an isomorphism for all $x \in I$.

Disjoint union axiom. (a) If the category $I=I_{1} \amalg I_{2}$ is the disjoint union of its full subcategories $I_{1}$ and $I_{2}$, then the corresponding inclusions $i_{1}, i_{2}$ of the subcategories $I_{1}$, $I_{2}$ into $I$ determine an equivalence of categories

$$
\left(i_{1}^{*}, i_{2}^{*}\right): \mathbf{C}_{I} \stackrel{\sim}{\longrightarrow} \mathbf{C}_{I_{1}} \times \mathbf{C}_{I_{2}} .
$$

(b) $\mathbf{C}_{\varnothing}$ is a trivial category (having precisely one morphism between any pair of objects).

Kan homotopy extension axioms. The Kan left homotopy extension axiom requires that for any functor $f: I \longrightarrow J$, the functor $f^{*}: \mathbf{C}_{J} \longrightarrow \mathbf{C}_{I}$ possesses a left adjoint $f_{!}: \mathbf{C}_{I} \longrightarrow \mathbf{C}_{J}$. By symmetry, the Kan right homotopy extension axiom says that $f^{*}$ has a right adjoint $f_{*}: \mathbf{C}_{I} \longrightarrow \mathbf{C}_{J}$. Below we shall also refer to the functors $f_{!}$and $f_{*}$ as to the left and right Kan homotopy extensions, respectively.

In the special case where $f: I^{\star} \longrightarrow 0^{\star}$ comes from a unique functor $I \longrightarrow 0$, we shall

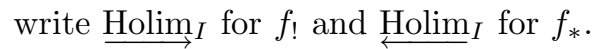

Lemma 1.2. Let $(f, g)$ be a pair of adjoint functors in $\mathcal{D i a}^{\star}$, and let $\varphi: f g \longrightarrow 1$, $\psi: 1 \longrightarrow g f$ be the adjunction morphisms. Then $\left(g^{*}, f^{*}\right)$ is a pair of adjoint functors and $\varphi^{*}: g^{*} f^{*} \longrightarrow 1, \psi^{*}: 1 \longrightarrow f^{*} g^{*}$ are the adjunction morphisms.

Proof. Straightforward.

Definition. The functor (1) is called a left (respectively, right) system of diagram categories if the above axioms (the functoriality axiom, the isomorphism axiom, the disjoint union axiom, and the left (respectively, right) Kan homotopy extension axiom) are satisfied.

In what follows, a left and right system of diagram categories is called a bisystem of diagram categories.

Example. Let $\mathcal{C}$ be a closed model category, and let $I \in$ Dirf. There is a natural structure of a closed model category for $\mathcal{C}^{I}$ (see [5]). Next, suppose that $\mathcal{C}$ has a zero object. Denote by $\mathrm{Ho}^{I}$ the homotopy category obtained by inverting the weak equivalences. There is a canonical functor $\mathcal{C}^{I} \longrightarrow \mathcal{C}^{I^{\star}}$ that extends an $I$-diagram to $I^{\star}$. It sends the zero object and morphisms in $I^{\star}$ to the zero object and morphisms in $\mathcal{C}$. Therefore, any functor $f: I^{\star} \longrightarrow J^{\star}$ yields a functor $f^{*}: \mathcal{C}^{J} \longrightarrow \mathcal{C}^{I}$. Since it preserves weak equivalences, it gives rise to a functor between homotopy categories. By [5, Subsection 1.3.2], the functor

$$
I \in \operatorname{Dirf} \mapsto \mathrm{HoC}^{I}
$$

determines a bisystem of diagram categories of the domain Dirf. 
Given an arbitrary model category $\mathcal{C}$, let $\mathcal{C}_{*}$ denote the model category under the terminal object $*$ (see $[13$, p. 4$]$ ). Then $\mathcal{C}_{*}$ is pointed. By definition, its bisystem of diagram categories with domain Dirf is the bisystem associated with $\mathcal{C}_{*}$.

Let $F: \mathbf{A} \longrightarrow \mathbf{C}$ be a morphism between two left systems $\mathbf{A}$ and $\mathbf{C}$ of diagram categories, and let $f: I \longrightarrow J$ be a map in $\mathcal{D i a}^{\star}$. Consider the adjunction maps

$$
\alpha: 1 \longrightarrow f^{*} f_{!} \text {and } \beta: f_{!} f^{*} \longrightarrow 1 \text {. }
$$

Let $\gamma_{F, f}$ denote the composed map

$$
f_{!} F \stackrel{f_{!} F \alpha}{\longrightarrow} f_{!} F f^{*} f_{!} \stackrel{f_{!} \iota_{F, f}^{-1} f_{!}}{\longrightarrow} f_{!} f^{*} F f_{!} \stackrel{\beta F f_{!}}{\longrightarrow} F f_{!} .
$$

We say that $F$ is right exact if $\gamma_{F, f}$ is an isomorphism and the following two compatibility relations are fulfilled:

$$
F \alpha_{\mathbf{A}}=\iota_{F, f} f_{!} \circ f^{*}\left(\gamma_{F, f}\right) \circ \alpha_{\mathbf{C}} F \quad \text { and } \quad F \beta_{\mathbf{A}}=\beta_{\mathbf{C}} F \circ f_{!}\left(\iota_{F, f}^{-1}\right) \circ \gamma_{F, f}^{-1} f^{*} .
$$

That is, $F \alpha_{\mathbf{A}}$ is the composition

$$
F \stackrel{\alpha_{\mathbf{C}} F}{\longrightarrow} f^{*} f_{!} F \stackrel{f^{*}\left(\gamma_{F, f}\right)}{\longrightarrow} f^{*} F f_{!} \stackrel{\iota_{F, f} f_{!}}{\longrightarrow} F f^{*} f_{!},
$$

and $F \beta_{\mathbf{A}}$ is the composition

$$
F f_{!} f^{*} \stackrel{\gamma_{F, f}^{-1} f^{*}}{\longrightarrow} f_{!} F f^{*} \stackrel{f_{!}\left(\iota_{F, f}^{-1}\right)}{\longrightarrow} f_{!} f^{*} F \stackrel{\beta_{\mathbf{C}} F}{\longrightarrow} F .
$$

The notion of a left exact (respectively, exact) morphism between two right systems of diagram categories (respectively, between two bisystems of diagram categories) is defined similarly.

Proposition 1.3. Let $F: \mathbf{A} \longrightarrow \mathbf{C}$ and $G: \mathbf{B} \longrightarrow \mathbf{C}$ be two right exact (respectively, left exact) morphisms between left systems of diagram categories (respectively, between right systems of diagram categories); then the fiber product $\prod(F, G)$ is also a left system of diagram categories (respectively, a right system of diagram categories).

Proof. It suffices to consider left systems of diagram categories. The case of a right system is proved by symmetry. By Proposition $1.1, \prod(F, G)$ is a presystem of diagram categories. Obviously, it satisfies both the isomorphism axiom and the disjoint union axiom. Thus, we must verify the Kan homotopy extension axioms.

Let $f: I \longrightarrow J$ be a map in $\mathcal{D i a}^{\star}$. We define a functor

$$
f_{!}: \prod(F, G)_{I} \longrightarrow \prod(F, G)_{J}
$$

as follows: $(A, c, B) \mapsto\left(f_{!}(A), \gamma_{G, f} f_{!}(c) \gamma_{F, f}^{-1}, f_{!}(B)\right)$. Then the adjunction maps $\alpha_{\mathbf{A}, \mathbf{B}}$ : $1 \longrightarrow f^{*} f_{!}$and $\beta_{\mathbf{A}, \mathbf{B}}: f_{!} f^{*} \longrightarrow 1$ determine adjunction maps for $\prod(F, G)$. To check this, we need to check that the squares

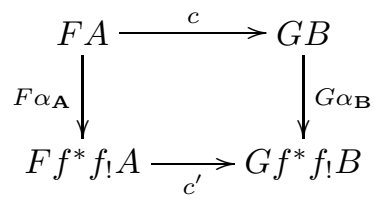

with $c^{\prime}=\iota_{G, f} f ! \circ f^{*}\left(\gamma_{G, f}\right) \circ f^{*} f_{!}(c) \circ f^{*}\left(\gamma_{F, f}^{-1}\right) \circ \iota_{F, f}^{-1} f_{!}$and

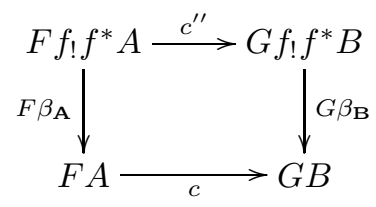


with $c^{\prime \prime}=\gamma_{G, f} f^{*} \circ f_{!}\left(\iota_{G, f}\right) \circ f_{!} f^{*}(c) \circ f_{!}\left(\iota_{F, f}^{-1}\right) \circ \gamma_{F, f}^{-1} f^{*}$ are commutative.

We have the following commutative diagram:

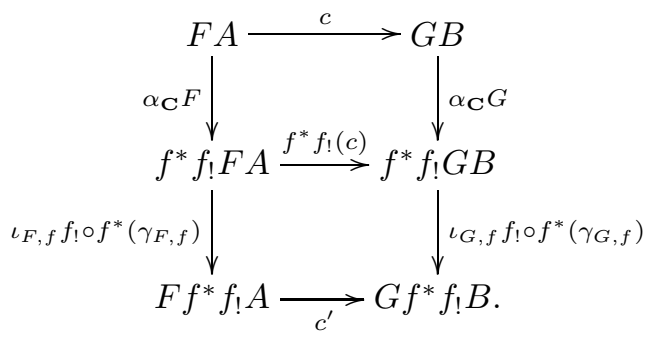

By (3), we obtain

$$
\begin{aligned}
G \alpha_{\mathbf{B}} \circ c & =\iota_{G, f} f_{!} \circ f^{*}\left(\gamma_{G, f}\right) \circ \alpha_{\mathbf{C}} G \circ c=\iota_{G, f} f_{!} \circ f^{*}\left(\gamma_{G, f}\right) \circ f^{*} f_{!}(c) \circ \alpha_{\mathbf{C}} F \\
& =\iota_{G, f} f_{!} \circ f^{*}\left(\gamma_{G, f}\right) \circ f^{*} f_{!}(c) \circ f^{*}\left(\gamma_{F, f}^{-1}\right) \circ \iota_{F, f}^{-1} f_{!} \circ F \alpha_{\mathbf{A}}=c^{\prime} \circ F \alpha_{\mathbf{A}} .
\end{aligned}
$$

Thus, the first square is commutative. The commutativity of the second square is checked in a similar way. It is routine to verify that the two compositions

$$
f^{*} \stackrel{\alpha f^{*}}{\longrightarrow} f^{*} f_{!} f^{*} \stackrel{f^{*} \beta}{\longrightarrow} f^{*}, \quad f_{!} \stackrel{f_{!} \alpha}{\longrightarrow} f_{!} f^{*} f_{!} \stackrel{\beta f_{!}}{\longrightarrow} f_{!}
$$

are identities (for $f_{\prod(F, G)}^{*}$ or, respectively, for $f_{!} \prod(F, G)$ ). This shows that the Kan homotopy extension axiom is fulfilled.

1.3. Consequences of axioms. In this subsection we discuss some consequences of the axioms. We also refer the reader to Franke's paper [5].

1.3.1. Properties of the Kan homotopy extension functors. A map $f: I \longrightarrow J$ in Dia is a closed (open) immersion if it is a fully faithful inclusion such that, for any $x \in J$, if $\operatorname{Hom}(I, x) \neq \varnothing(\operatorname{Hom}(x, I) \neq \varnothing)$, then $x \in I$. The following is straightforward.

Immersion lemma. Let $f: I \longrightarrow J$ be a closed (respectively, open) immersion in Dia. Then the map $g: J^{\star} \longrightarrow I^{\star}$ that takes $j \in J$ to $j$ if $j \in I$ and to $\star$ otherwise is a right (respectively, left) adjoint to $f^{\star}$.

Proposition 1.4. Suppose $\mathbf{C}$ is a left system of diagram categories. Let $f: I \longrightarrow J$ be a functor, let $x \in J$, and let $i_{x}: J / x \longrightarrow J, j_{x}: f / x \longrightarrow I$, and $l: f / x \longrightarrow J / x$ be the canonical functors. If $J$ is a poset, then for $A \in \mathbf{C}_{I}$ we have isomorphisms

$$
\left(f_{!} A\right)_{x} \simeq \underline{\operatorname{Holim}}_{J / x} i_{x}^{*} f_{!} A \simeq \underline{\operatorname{Holim}}_{J / x} l_{!} j_{x}^{*} A \simeq \underline{\operatorname{Holim}}_{f / x} j_{x}^{*} A .
$$

If $\mathbf{C}$ is a right system of diagram categories, a dual assertion is valid for projective homotopy limits and right Kan homotopy extensions.

Proof. The proof is similar to that in [5, Subsection 1.4.2].

If $f$ is the inclusion $I \subseteq J$ of a full subcategory, where $J$ is a poset, then for every object $x \in I$ the category $f / x$ has a final object $\left(x, \mathrm{id}_{x}\right)$. Therefore, the following statement is true.

Corollary 1.5. Let $\mathbf{C}$ be a left system of diagram categories (respectively, right system of diagram categories), and let $f: I \longrightarrow J$ be the inclusion of a full subcategory, where $J$ is a poset. Then the canonical morphism $A \longrightarrow f^{*} f_{!} A$ in $\mathbf{C}_{I}$ (respectively, $f^{*} f_{*} A \longrightarrow A$ ) is an isomorphism for every object $A \in \mathbf{C}_{I}$.

Proposition 1.4 is often employed to reduce assertions about the functors $f_{!}$and $f_{*}$ to similar assertions about $\underline{\operatorname{Holim}}_{J}$ and $\stackrel{\operatorname{Holim}}{J}_{J}$. The following proposition is concerned with the replacement of $J$ by a smaller category. 
Proposition 1.6. Suppose $\mathbf{C}$ is a left system of diagram categories. Let $i: I^{\star} \longrightarrow J^{\star}$ be some functor (typically, the inclusion of a subcategory). If $i$ has a left adjoint of the form $l^{\star}$ with $l: J \longrightarrow I$, then $\underline{\operatorname{Holim}}_{J} A \simeq \underline{\operatorname{Holim}}_{I} i^{*} A$. Dually, suppose $\mathbf{C}$ is a right system of

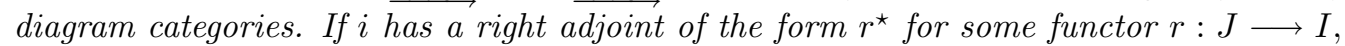
then $\operatorname{Holim}_{J} A \simeq \operatorname{Holim}_{I} i^{*} A$.

Proof. (1) Let $l$ be a left adjoint of $i$. Then $l_{\text {! }}$ is naturally isomorphic to $i^{*}$. It follows that $\underline{\operatorname{Holim}}_{J}=\underline{\operatorname{Holim}}_{I} \circ l_{!} \simeq \underline{\operatorname{Holim}}_{I} \circ i^{*}$.

1.3.2. Cartesian squares. Let $\square \in \mathcal{D}$ ia be the poset $\Delta^{1} \times \Delta^{1}$ possessing the following elements:

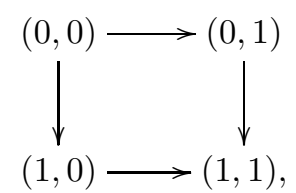

where $\longrightarrow$ stands for $<$. Let $\ulcorner\subset \square$ be the subposet obtained by removing the lower right corner $(1,1)$, and let $\lrcorner \subset \square$ be the subposet containing all elements except for $(0,0)$. Let $i_{\ulcorner}:\left\ulcorner\longrightarrow \square\right.$ and $\left.i_{\lrcorner}:\right\lrcorner \longrightarrow \square$ be inclusions. Let $\mathbf{C}$ be a right system of diagram categories (respectively, a left system of diagram categories). An object $A$ of $\mathbf{C}_{\square}$ is said to be Cartesian (respectively, co-Cartesian) if and only if the canonical morphism $A \longrightarrow i_{\lrcorner *} i_{\lrcorner}^{*} A$ is an isomorphism (respectively if and only if the canonical morphism $i_{\ulcorner!} i_{\ulcorner}^{*} A \longrightarrow A$ is an isomorphism).

Lemma 1.7. Let $\mathbf{C}$ be a left system of diagram categories. An object $A$ of $\mathbf{C}_{\square}$ is coCartesian if and only if $A_{(1,1)} \simeq \operatorname{Holim}_{\ulcorner} i_{\ulcorner}^{*} A$. Dually, if $\mathbf{C}$ is a right system of diagram categories, then an object $A$ of $\mathbf{C}_{\square}$ is Cartesian if and only if $A_{(0,0)} \simeq \underset{\text { Holim}}{\longleftarrow} i_{\lrcorner}^{*} A$.

Proof. Suppose $\mathbf{C}$ is a left system of diagram categories. Let $A$ be an arbitrary object of $\mathbf{C}_{\square}$. By Corollary 1.5, the natural morphism

$$
i_{\ulcorner}^{*} A \longrightarrow i_{\ulcorner}^{*} i_{\ulcorner!} i_{\ulcorner}^{*} A
$$

is an isomorphism. Therefore, $A_{(i, j)} \simeq i_{\ulcorner!} i_{\ulcorner}^{*} A_{(i, j)}$ if $(i, j) \in\{(0,0),(0,1),(1,0)\}$.

Proposition 1.4 shows that $i_{\ulcorner!} i_{\ulcorner}^{*} A_{(1,1)} \simeq \underline{\text { Holim}}_{\ulcorner} i_{\ulcorner}^{*} A$, and the claim follows.

From now on, all left or right systems of diagram categories are assumed to be systems with domain $\mathcal{O} \mathrm{rd}^{\star}$. An object $A$ of $\mathbf{C}_{I \times \square}, I \in \mathrm{Ord}$, is said to be Cartesian if

$$
A \longrightarrow\left(\operatorname{id}_{I} \times i_{\lrcorner}\right)_{*}\left(\operatorname{id}_{I} \times i_{\lrcorner}\right)^{*} A
$$

is an isomorphism; the co-Cartesian objects are defined similarly, by replacing a right system $\mathbf{C}$ of diagram categories with a left system of diagram categories, $\lrcorner$ with $\ulcorner$, and $\left(\mathrm{id}_{I} \times i_{\lrcorner}\right)_{*}$ with $\left(\operatorname{id}_{I} \times i_{\ulcorner}\right)$!, and by reversing the direction of the arrow. The isomorphism axiom and Proposition 1.8 below imply that an object $A \in \mathbf{C}_{I \times \square}$ is Cartesian (respectively, co-Cartesian) if and only if the object $A_{x, \square}=\left(i_{x, I} \times \mathrm{id}_{\square}\right)^{*} A$ is Cartesian (respectively, co-Cartesian) in $\mathbf{C}_{\square}$ for all $x \in I$.

Let $\mathbf{C}$ be a left system of diagram categories. For any object $I$ of Ord, we denote by $\mathbf{C}(I)$ the left system of diagram categories defined as $\mathbf{C}(I)_{J}=\mathbf{C}_{I \times J}$. Here $I$ plays the role of a parameter.

Proposition 1.8. Let $\mathbf{C}$ be a left system of diagram categories, and let $f: I \longrightarrow J$ be a functor in $\mathcal{O r d}^{\star}$. Then $f$ gives rise to the right exact functor $f^{*}: \mathbf{C}(J) \longrightarrow \mathbf{C}(I)$ induced by $\left(f \times 1_{K}\right)^{*}: \mathbf{C}_{J \times K} \longrightarrow \mathbf{C}_{I \times K}$ with $K \in \mathcal{O} \mathrm{rd}^{\star}$. In particular, the functor $f^{*}$ respects the co-Cartesian squares. A dual assertion is also valid for right systems of diagram categories. 
Proof. For a map $g: K \longrightarrow L$ in $\mathcal{O r d}^{\star}$, we need to show that a natural morphism

$$
\gamma:\left(1_{I} \times g\right) !\left(f \times 1_{K}\right)^{*} \longrightarrow\left(f \times 1_{L}\right)^{*}\left(1_{J} \times g\right) !
$$

is an isomorphism. Suppose $A \in \mathbf{C}_{J \times K}$ and $(x, y) \in I \times L$.

The map $\varphi_{x, y}: 1 \times g /(x, y) \longrightarrow g / y,((u, u \longrightarrow x),(v, g(v) \longrightarrow y)) \longmapsto(v, g(v) \longrightarrow y)$, has a right adjoint $\psi_{x, y}: g / y \longrightarrow 1 \times g /(x, y),(v, g(v) \longrightarrow y) \longmapsto((x, x=x),(v, g(v) \longrightarrow$ $y)$ ). By Proposition 1.6, we have $\underline{\operatorname{Holim}}_{1 \times g /(x, y)} \simeq \underline{\operatorname{Holim}}_{g / y} \psi_{x, y}^{*}$.

Proposition 1.4 implies that

$$
\begin{aligned}
\left(1_{I} \times g\right)_{!}\left(f \times 1_{K}\right)^{*} A_{(x, y)} & \simeq \underline{\operatorname{Holim}}_{1_{I} \times g /(x, y)} j_{(x, y)}^{*}\left(f \times 1_{K}\right)^{*} A \\
& \simeq \stackrel{\operatorname{Holim}}{\longrightarrow} g / y_{x, y} \psi_{x, y)}^{*} j_{(x, y)}^{*}\left(f \times 1_{K}\right)^{*} A .
\end{aligned}
$$

On the other hand,

$$
\begin{gathered}
\left(f \times 1_{L}\right)^{*}\left(1_{J} \times g\right)_{!} A_{(x, y)}=i_{(f(x), y)}^{*}\left(1_{J} \times g\right) ! A \simeq \underline{\operatorname{Holim}}_{1_{J} \times g /(f(x), y)} j_{(f(x), y)}^{*} A \\
\simeq \stackrel{\operatorname{Holim}}{\longrightarrow} / y_{f} \psi_{f(x), y}^{*} j_{(f(x), y)}^{*} A=\underline{\operatorname{Holim}}_{g / y} \psi_{x, y}^{*} j_{(x, y)}^{*}\left(f \times 1_{K}\right)^{*} A .
\end{gathered}
$$

Here we have used the relation $j_{(f(x), y)} \psi_{f(x), y}=\left(f \times 1_{K}\right) j_{(x, y)} \psi_{x, y}$. Thus, $\gamma$ is an isomorphism. It is routine to check the compatibility relations (3).

Definition. Let $\mathbf{C}$ be a right (respectively, left) system of diagram categories. A square in $I$ is a functor $i: \square \longrightarrow I$ injective on the set of objects. Let $A$ be an object of $\mathbf{C}_{I}$; we say that $A$ makes the square $i$ Cartesian (respectively, co-Cartesian) if $i^{*} A$ is Cartesian (respectively, co-Cartesian).

Proposition 1.9. Let $\mathbf{C}$ be a left system of diagram categories, and let $i$ be a square in a poset $I$. If the functor $\ulcorner\longrightarrow(I-i(1,1) / i(1,1))$ possesses a left adjoint and if $A=f_{!} B$, where $f: J \longrightarrow I$ is a functor not containing $i(1,1)$ in its image, then $A$ makes $i$ co-Cartesian. A similar statement is valid if $\mathbf{C}$ is a right system of diagram categories, the functor $\lrcorner \longrightarrow(I-i(0,0) \backslash i(0,0))$ possesses a right adjoint, and $A=f_{*} B$, where $f: J \longrightarrow I$ is a functor not containing $i(0,0)$ in its image.

Proof. The proof is similar to that in [5, Subsection 1.4.5].

Proposition 1.10 (Concatenation of squares and the property of being (co-)Cartesian). Let $\mathbf{C}$ be a left (respectively, right) system of diagram categories, let $d_{0,1,2}: \Delta^{1} \longrightarrow \Delta^{2}$ be three monotone injections, and let $A \in \mathbf{C}_{\Delta^{2} \times \Delta^{1}}$. Suppose that $\left(d_{2} \times \operatorname{id}_{\Delta^{1}}\right)^{*} A \in \mathbf{C}_{\square}$ is co-Cartesian (respectively, $\left(d_{0} \times \mathrm{id}_{\Delta^{1}}\right)^{*} A \in \mathbf{C}_{\square}$ is Cartesian). Then $\left(d_{0} \times \operatorname{id}_{\Delta^{1}}\right)^{*} A$ is co-Cartesian (respectively, $\left(d_{2} \times \mathrm{id}_{\Delta^{1}}\right)^{*} A$ is Cartesian) if and only if $\left(d_{1} \times \mathrm{id}_{\Delta^{1}}\right)^{*} A$ is co-Cartesian (respectively, $\left(d_{1} \times \operatorname{id}_{\Delta^{1}}\right)^{*} A$ is Cartesian).

Proof. The proof is similar to that in [5, Subsection 1.4.6].

Proposition 1.11. Let $\mathbf{C}$ be a left (respectively, right) system of diagram categories. For every I, the category $\mathbf{C}_{I}$ has a zero object and finite coproducts (respectively, products). For every functor $f: I \longrightarrow J$, the functor $f_{!}$(respectively, $f_{*}$ ) preserves coproducts (respectively, products).

Proof. Let $f: I^{\star} \longrightarrow \varnothing^{\star}$ be the only functor. The inclusion $g: \varnothing^{\star} \longrightarrow I^{\star}$ is left and right adjoint to $f$. It follows that $g^{*}$ is left and right adjoint to $f^{*}$. Therefore, if $0 \in \mathbf{C}_{\varnothing}$, then the object $f^{*} 0$ (we denote it also by 0 ) is a zero object in $\mathbf{C}_{I}$.

Let $I \amalg I$ be the disjoint union of two copies of $I$, and let $p: I \amalg I \longrightarrow I$ be the functor that is the identity on both copies of $I$. By the disjoint union axiom, we have

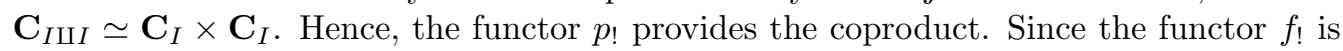
left adjoint to $f^{*}$, where $f: I \longrightarrow J$ is a map in $\mathcal{D}$ ia, it preserves coproducts. 
Let $f: I^{\star} \longrightarrow J^{\star}$ be a map in $\mathcal{D i a}{ }^{\star}$, and let $x \in I$. If $f(x)=\star$, then $f^{*} A_{x}=$ $i_{x, I}^{*} f^{*} A=0$ for any $A \in \mathbf{C}_{J}$. Indeed, the composition $0^{\star} \stackrel{i_{x, I}}{\longrightarrow} I^{\star} \stackrel{f}{\longrightarrow} J^{\star}$ factors as $0^{\star} \stackrel{j}{\longrightarrow} \varnothing^{\star} \stackrel{l}{\longrightarrow} J^{\star}$, whence $f^{*} A_{x}=j^{*} l^{*} A=0$.

\section{§2. DÉRIVATEurS}

2.1. Definitions. Let $\mathcal{D}$ ia be a category of diagrams. So far, we considered only functors

$$
\text { C : } \text { Dia }^{\star \text { op }} \longrightarrow \text { CAT }
$$

evaluated on the category $\mathcal{D}$ ia ${ }^{\star}$. The horizontal morphisms $I \longrightarrow J$ in $\mathcal{D}$ ia ${ }^{\star}$ are given by the functors $I^{\star} \longrightarrow J^{\star}$ mapping $\star$ to $\star$. Of particular interest are the functors

$$
\mathbf{D}: \mathrm{Dia}^{\mathrm{op}} \longrightarrow \mathbf{C A T}
$$

evaluated on the underlying category $\mathcal{D}$ ia. Here we follow the terminology of [7].

All the axioms of $\S 1$ can also be reformulated for morphisms and bimorphisms in $\mathcal{D}$ ia.

Definition. A functor of the form (4) is called a prédérivateur if it satisfies the functoriality axiom. It is a left (respectively, right) dérivateur if it satisfies the functoriality axiom, the isomorphism axiom, the disjoint union axiom, the left (respectively, right) Kan homotopy extension axiom, and the left (respectively, right) base change axiom (see below).

Base change axiom. Let $f: I \longrightarrow J$ be a morphism in $\mathcal{D}$ ia, and let $x \in J$. Consider the following diagram in $\mathcal{D}$ ia:

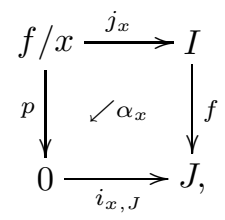

where $j_{x}$ is a natural map and $\alpha_{x}$ is the bimorphism

$$
f j_{x} \longrightarrow i_{x, I} p, \quad \alpha_{x}: f j_{x}(y, a: f(y) \longrightarrow x)=f(y) \stackrel{a}{\longrightarrow} x=i_{x, J} p(y, a) .
$$

The bimorphism $\alpha_{x}$ induces a bimorphism $\beta_{x}: p_{!} j_{x}^{*} \longrightarrow i_{x, I}^{*} f_{!}$, namely, the composition

$$
p_{!} j_{x}^{*} \longrightarrow p_{!} j_{x}^{*} f^{*} f_{!} \stackrel{p_{!} \alpha_{x}^{*} f_{!}}{\longrightarrow} p_{!} p^{*} i_{x}^{*} f_{!} \longrightarrow i_{x}^{*} f_{!} \cdot
$$

The left base change axiom requires that $\beta_{x}$ be an isomorphism.

Symmetrically, the right base change axiom says that the diagram

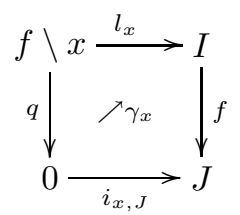

yields an isomorphism $\delta_{x}: i_{x, I}^{*} f_{*} \longrightarrow q_{*} l_{x}^{*}$.

The left and right dérivateurs will be called bidérivateurs.

Example. If a category $\mathcal{C}$ is closed under colimits, then the representable prédérivateur associated with it is a left dérivateur. A typical example of a bidérivateur (with domain Dirf) is given by the functor $I \mapsto \operatorname{HoC}^{I}$ with $\mathcal{C}$ a closed model category (see [14] for details). 
In what follows, all left or right dérivateurs are assumed to have a fixed domain $\mathcal{D}$ ia. The morphisms between two prédérivateurs and the fiber product of a pair of morphisms are defined by analogy with the case of presystems of diagram categories. As before, it can be proved that the fiber product of two morphisms is a prédérivateur, which is a left (right) dérivateur whenever both morphisms are right (left) exact.

Proposition 2.1. Suppose $\mathbf{D}$ is a left dérivateur. Let $f: I \longrightarrow J$ be a functor in Dia, and let $x \in J$. Then for $A \in \mathbf{D}_{I}$ we have the isomorphism

$$
\left(f_{!} A\right)_{x} \simeq \underline{\operatorname{Holim}}_{f / x} j_{x}^{*} A .
$$

If $\mathbf{D}$ is a right dérivateur, then a dual assertion is valid for the projective homotopy limits and the right Kan homotopy extensions.

Proof. Straightforward.

If $f$ is the inclusion $I \subseteq J$ of a full subcategory, then for every object $x \in I$ the category $f / x$ has a final object $\left(x, \mathrm{id}_{x}\right)$. This leads to the following statement.

Corollary 2.2. Let $\mathbf{D}$ be a left (respectively, right) dérivateur, and let $f: I \longrightarrow J$ be the inclusion of a full subcategory. Then the canonical morphism $A \longrightarrow f^{*} f_{!} A$ (respectively, $\left.f^{*} f_{*} A \longrightarrow A\right)$ in $\mathbf{D}_{I}$ is an isomorphism for every object $A$ of $\mathbf{D}_{I}$.

The notion of a (co-)Cartesian square is defined as before. Below we formulate statements about (co-)Cartesian squares without proof. The proofs repeat those in the preceding section word for word.

Lemma 2.3. Let $\mathbf{D}$ be a left dérivateur. An object $A$ of $\mathbf{D}_{\square}$ is co-Cartesian if and only if $A_{(1,1)} \simeq \underline{\text { Holim }}_{\ulcorner} i_{\ulcorner}^{*} A$. Dually, if $\mathbf{D}$ is a right dérivateur, then an object $A$ of $\mathbf{D}_{\square}$ is

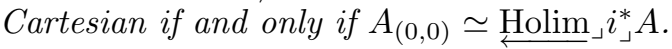

For any object $I$ of $\mathcal{D}$ ia, we denote by $\mathbf{D}(I)$ the left dérivateur defined by the rule $\mathbf{D}(I)_{J}=\mathbf{D}_{I \times J}$.

Proposition 2.4. Let $\mathbf{D}$ be a left dérivateur. Let $f: I \longrightarrow J$ be a map in Dia. Then $f$ gives rise to the right exact functor $f^{*}: \mathbf{D}(J) \longrightarrow \mathbf{D}(I)$ induced by $\left(f \times 1_{K}\right)^{*}$ : $\mathbf{D}_{J \times K} \longrightarrow \mathbf{D}_{I \times K}$ with $K \in \mathcal{D}$ ia. In particular, $f^{*}$ respects the co-Cartesian squares. A dual assertion is also valid for the right dérivateurs.

Proposition 2.5. Let $\mathbf{D}$ be a left dérivateur, and let $i$ be a square in $I \in \mathcal{D}$ ia. If the functor $\left\ulcorner\longrightarrow(I-i(1,1) / i(1,1))\right.$ possesses a left adjoint and $A=f_{!} B$, where $f: J \longrightarrow I$ is a functor not containing $i(1,1)$ in its image, then $A$ makes $i$ co-Cartesian. Let $\mathbf{D}$ be a right dérivateur. A similar statement is true if the functor $\lrcorner \longrightarrow(I-i(0,0) \backslash i(0,0))$ possesses a right adjoint and $A=f_{*} B$, where $f: J \longrightarrow I$ is a functor not containing $i(0,0)$ in its image.

Proposition 2.6 (Concatenation of squares and the property of being (co-)Cartesian). Let $\mathbf{D}$ be a left (respectively, right) dérivateur, let $d_{0,1,2}: \Delta^{1} \longrightarrow \Delta^{2}$ be three monotone injections, and let $A \in \mathbf{D}_{\Delta^{2} \times \Delta^{1}}$. Suppose that $\left(d_{2} \times \mathrm{id}_{\Delta^{1}}\right)^{*} A \in \mathbf{D}_{\square}$ is co-Cartesian (respectively, $\left(d_{0} \times \operatorname{id}_{\Delta^{1}}\right)^{*} A \in \mathbf{D}_{\square}$ is Cartesian). Then $\left(d_{0} \times \operatorname{id}_{\Delta^{1}}\right)^{*} A$ is co-Cartesian (respectively, $\left(d_{2} \times \operatorname{id}_{\Delta^{1}}\right)^{*} A \in \mathbf{D}_{\square}$ is Cartesian) if and only if $\left(d_{1} \times \mathrm{id}_{\Delta^{1}}\right)^{*} A$ is coCartesian (respectively, $\left(d_{1} \times \mathrm{id}_{\Delta^{1}}\right)^{*} A \in \mathbf{D}_{\square}$ is Cartesian).

Proposition 2.7. Let $\mathbf{D}$ be a left (respectively, right) dérivateur. For every $I$, the category $\mathbf{D}_{I}$ has an initial (respectively, final) object and finite coproducts (respectively, products). For every functor $f: I \longrightarrow J$, the functor $f_{!}$(respectively, $f_{*}$ ) preserves coproducts (respectively, products). 
Proof. Let $f: \varnothing \longrightarrow I$ be the inclusion, and let $0 \in \mathbf{D}_{\varnothing}$. Since $f_{\text {! }}$ is a left adjoint to $f^{*}$, it follows that $f_{!} 0$ (we denote it also by 0 ) is an initial object in $\mathbf{D}_{I}$.

Let $I \amalg I$ be the disjoint union of two copies of $I$, and let $p: I \amalg I \longrightarrow I$ be the functor that is the identity on both copies of $I$. By the disjoint union axiom, we have

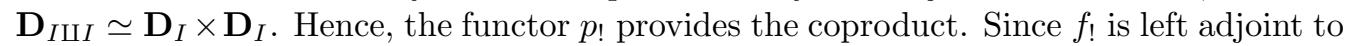
$f^{*}$, where $f: I \longrightarrow J$ is a map in $\mathcal{D}$ ia, $f$ ! preserves coproducts and $f^{*}$ preserves products (whenever they exist).

2.2. Pointed dérivateurs. The dérivateurs to be dealt with below will satisfy some additional conditions. We start with definitions.

Definition. A left dérivateur is said to be pointed if the following three conditions are satisfied:

(1) for any closed immersion $f: I \longrightarrow J$ in $\mathcal{D}$ ia, the structure functor $f_{\text {! }}$ possesses a left adjoint $f^{\text {? }}$;

(2) for any open immersion $f: I \longrightarrow J$ in $\mathcal{D}$ ia, the structure functor $f^{*}$ possesses a right adjoint $f_{*}$;

(3) for any open immersion $f: I \longrightarrow J$ in $\mathcal{D}$ ia and any object $x \in J$, the base change morphism of the diagram

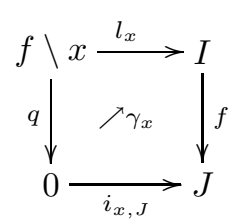

yields an isomorphism $\delta_{x}: i_{x, I}^{*} f_{*} \longrightarrow q_{*} l_{x}^{*}$. The corresponding notion of a pointed right dérivateur is defined in a similar way.

We note that for any open immersion $f: I \longrightarrow J$ in $\mathcal{D}$ ia and any object $x \in J$, a right adjoint $q_{*}$, where $q: f \backslash x \longrightarrow 0$ is a unique map, always exists. Indeed, if $x$ is not in $I$, then $f \backslash x=\varnothing$, and $q_{*}$ exists because $\varnothing \longrightarrow 0$ is an open immersion. If $x \in I$, then $f \backslash x$ has an initial object $(x, x=x)$, and we put $q_{*}=p^{*}$, where $0 \stackrel{p}{\longmapsto}(x, x=x) \in f \backslash x$.

Let $\mathbf{D}$ be a left (right) pointed dérivateur. Then $\mathbf{D}_{I}$ has a zero object for any $I \in \mathcal{D}$ ia, because the inclusion $\varnothing \longrightarrow I$ is a closed and open immersion, whence $0=f_{!} 0\left(0=f_{*} 0\right)$ is also a final (initial) object. Also, it follows that, for every open immersion $f: I \longrightarrow J$ in $\mathcal{D}$ ia and any object $x \in J$, the "value" $f_{*} A_{x}$ at $x, A \in \mathbf{D}_{I}$, is equal to $A_{x}$ if $x \in I$ and to 0 otherwise.

In what follows, a left and right pointed dérivateur will be referred to as a pointed bidérivateur.

2.3. Example. If $I \in \operatorname{Dirf}$ and $\mathcal{C}$ is a Waldhausen category $\mathcal{C}$, then the functor category $\mathcal{C}^{I}$ is also a Waldhausen category. A map $F \longrightarrow G$ in $\mathcal{C}^{I}$ is a cofibration (respectively, weak equivalence) if so is $F(x) \longrightarrow G(x)$ for every $x \in I$.

Definition. Let $\mathcal{A}$ be a category with finite coproducts and an initial object $e$. Assume that $\mathcal{A}$ has two distinguished classes of maps, called weak equivalences and cofibrations. A map is called a trivial cofibration if it is simultaneously a weak equivalence and a cofibration. Following Brown [15], we call $\mathcal{A}$ a category of cofibrant objects if the following axioms are satisfied.

(A) Let $f$ and $g$ be maps such that $g f$ is defined. If two of $f, g, g f$ are weak equivalences, then so is the third. Any isomorphism is a weak equivalence.

(B) The composition of two cofibrations is a cofibration. Any isomorphism is a cofibration. 
(C) Given a diagram

$$
A \stackrel{u}{\longleftarrow} C \stackrel{v}{\longrightarrow} B
$$

with $v$ a cofibration (respectively, a trivial cofibration), the pushout $A \amalg_{C} B$ exists and the map $A \longrightarrow A \amalg_{C} B$ is a cofibration (respectively, a trivial cofibration).

(D) Any map $u$ in $\mathcal{A}$ can be factored as $u=p i$, where $p$ is a weak equivalence and $i$ is a cofibration.

(E) For any object $A$, the map $e \longrightarrow A$ is a cofibration.

For instance, the Waldhausen category of bounded complexes $C^{b}(\mathcal{E})$ of an exact category $\mathcal{E}$ in which the weak equivalences are quasiisomorphisms and the cofibrations are componentwise admissible monomorphisms is a category of cofibrant objects.

Let $\mathcal{C}$ be a Waldhausen category of cofibrant objects, and let $\mathrm{Ho} \mathcal{C}$ denote the category obtained from $\mathcal{C}$ by inverting weak equivalences. The notion of a homotopy for two maps $f$ and $g$ can be introduced (see [15]). Consider the category $\pi \mathcal{C}$ with the same objects as in $\mathcal{C}$ and with $\pi \mathcal{C}(A, B)$ equal to the quotient of $\mathcal{C}(A, B)$ by the equivalence relation $f \sim g$ defined in terms of the homotopy. Then the class of weak equivalences in $\pi \mathcal{C}$ admits calculation of left fractions [15]. If $I \in$ Dirf, then, by [16, Subsection 1.31], the functor category $\mathcal{C}^{I}$ is a Waldhausen category of cofibrant objects.

Theorem 2.8 (Cisinski [16]). If $\mathcal{C}$ is a Waldhausen category of cofibrant objects, then the hyperfunctor

$$
\mathrm{DC}: I \in \operatorname{Dirf} \longmapsto \mathbf{D} \mathcal{C}_{I}=\mathrm{Ho}^{I}
$$

determines a left pointed dérivateur with domain Dirf.

\section{$\S 3$. The S.-CONSTRUCTION}

Throughout this section, $\mathbf{B}$ is assumed to be either a left system of diagram categories (with domain $\mathcal{O} \mathrm{rd}^{\star}$ ) or a left pointed dérivateur (with domain $\mathcal{D}$ ia). Let $\operatorname{Ar} \Delta^{n}$ be the poset of pairs $(i, j), 0 \leq i \leq j \leq n$, where $(i, j) \leq\left(i^{\prime}, j^{\prime}\right)$ if and only if $i \leq i^{\prime}$ and $j \leq j^{\prime}$. Regarded as a category, this poset can be identified with the category of arrows of $\Delta^{n}$.

For $0 \leq i<j<k \leq n$, let

$$
a_{i, j, k}: \square \longrightarrow \operatorname{Ar} \Delta^{n}
$$

be defined by the rule

$$
(0,0) \mapsto(i, j), \quad(0,1) \mapsto(i, k), \quad(1,0) \mapsto(j, j), \quad(1,1) \mapsto(j, k) .
$$

For $n \geq 0$, we denote by $S_{n} \mathbf{B}$ the full subcategory of $\mathbf{B}_{\mathrm{Ar} \Delta^{n}}$ consisting of the following objects $X$ :

$\diamond$ for any $i \leq n$, the object $X_{(i, i)}$ is isomorphic to zero in $\mathbf{B}_{0}$;

$\diamond$ if $n>1$, then for any $0 \leq i<j<k \leq n$ the square $a_{i, j, k}^{*} X$ is co-Cartesian.

The definition of $S_{n} \mathbf{B}$ is similar to that of $S_{n} \mathcal{C}$, where $\mathcal{C}$ is a Waldhausen category (see [9] for the details). Note that $S_{0} \mathbf{B}$ is the full subcategory of zero objects in $\mathbf{B}_{0}$. The category $S_{1} \mathbf{B}$ consists of the objects $X \in \mathbf{B}_{\Delta^{2}}$ such that $X_{0}$ and $X_{2}$ are isomorphic to zero.

Proposition 3.1. Let $n \geq 1$, and let $\ell: \Delta^{n-1} \longrightarrow \operatorname{Ar} \Delta^{n}$ be the map that takes $j$ to $(0, j+1)$. Then the functor $\ell^{*}$ induces an equivalence of the categories $S_{n} \mathbf{B}$ and $\mathbf{B}_{\Delta^{n-1}}$.

Proof. The proof splits into two steps.

I. We consider the left systems of diagram categories and the left pointed dérivateurs separately. 
(a) Suppose $\mathbf{B}$ is a left system of diagram categories. Consider the following full subcategory $I$ of $\operatorname{Ar} \Delta^{n}$ :

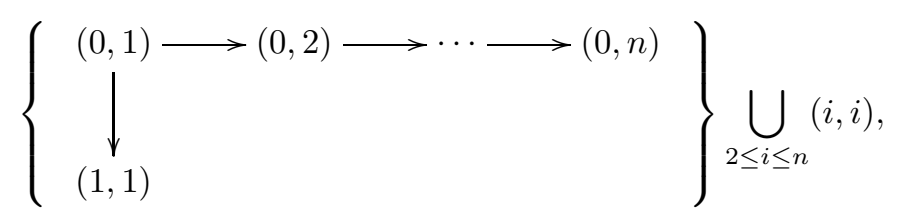

together with the map $g: \Delta^{n-1} \longrightarrow I, j \mapsto(0, j+1)$. Since $g$ is an open immersion, the immersion lemma shows that $g$ possesses the left adjoint $f: I^{\star} \longrightarrow \Delta^{n-1 \star},(0, j) \longmapsto j-1$ and $(i, i) \longmapsto \star$. Hence, $f^{*}$ is a right adjoint to $g^{*}$.

Let $\widetilde{\mathbf{B}}_{I}$ denote the full subcategory of $\mathbf{B}_{I}$ consisting of the objects $X \in \mathbf{B}_{I}$ such that the $X_{(i, i)}, 1 \leq i \leq n$, are isomorphic to zero. We claim that $f^{*}$ and $g^{*}$ are mutually inverse equivalences between $\mathbf{B}_{\Delta^{n-1}}$ and $\widetilde{\mathbf{B}}_{I}$. Indeed, $f^{*} A \in \widetilde{\mathbf{B}}_{I}$ for any $A \in \mathbf{B}_{\Delta^{n-1}}$, and $g^{*} f^{*}=1$. On the other hand, the adjunction map $B \longrightarrow f^{*} g^{*} B$ is an isomorphism for every $B \in \widetilde{\mathbf{B}}_{I}$.

(b) Suppose $\mathbf{B}$ is a left pointed dérivateur. Since $g$ is an open immersion, the functor $g^{*}$ possesses a right adjoint $g_{*}$. We show that $g^{*}$ and $g_{*}$ are mutually inverse equivalences between $\mathbf{B}_{\Delta^{n-1}}$ and $\widetilde{\mathbf{B}}_{I}$. Indeed, the adjunction map $g^{*} g_{*} \longrightarrow 1$ is an isomorphism by Corollary 2.2. Since $g$ is an open immersion, we see that $g_{*} B$ is in $\widetilde{\mathbf{B}}_{I}$ for all $B \in \mathbf{B}_{\Delta^{n-1}}$ (see the corresponding remarks on p. 968). It follows immediately that the adjunction map $B \longrightarrow g_{*} g^{*} B$ is an isomorphism for every $B \in \widetilde{\mathbf{B}}_{I}$.

II. Now, let $h: I \longrightarrow \operatorname{Ar} \Delta^{n}$ be an inclusion. Propositions 1.9 and 2.5 imply that for every $A \in \mathbf{B}_{I}$ the object $h_{!} A$ makes all squares

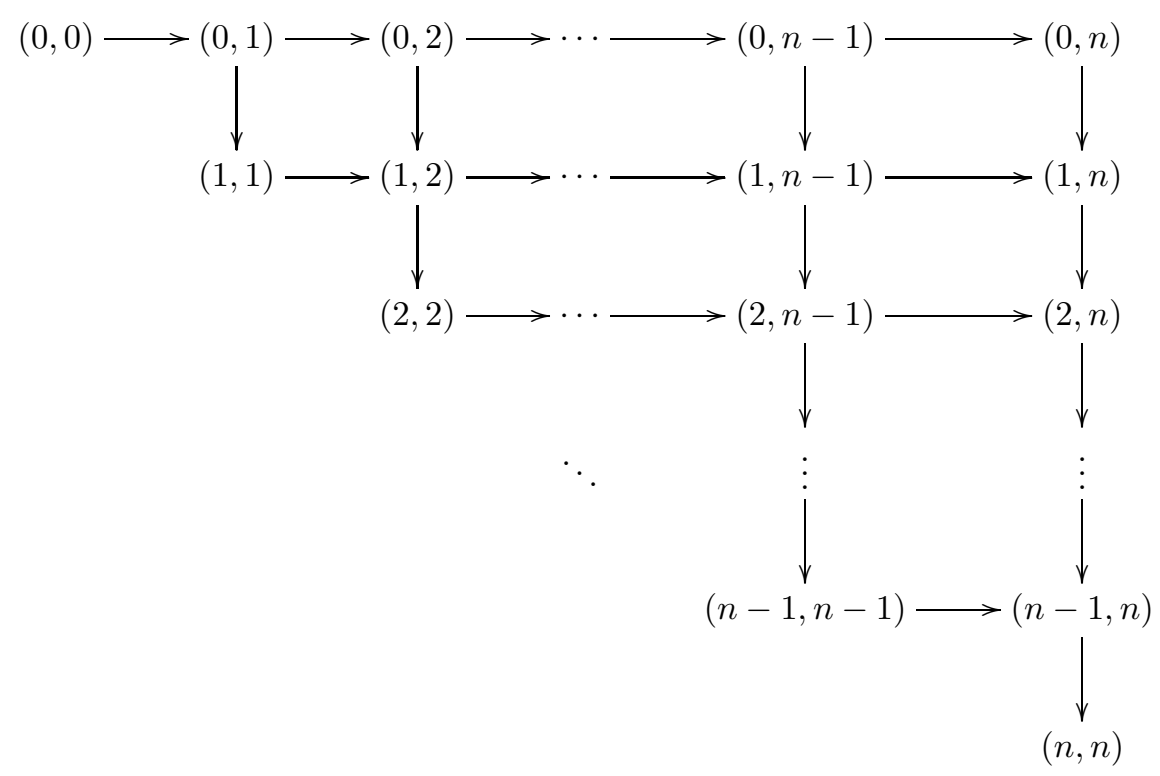

in the category $\operatorname{Ar} \Delta^{n}$ co-Cartesian. By Propositions 1.10 and 2.6, the same is true for all concatenations of squares.

Propositions 1.4 and 2.1 show that $h_{!} A_{(0,0)}$ is isomorphic to zero. By Corollaries 1.5 and 2.2, the canonical morphism $A \longrightarrow h^{*} h_{!} A$ is an isomorphism for all $A \in \mathbf{B}_{I}$. Let $1 \leq i \leq n$; then

$$
0 \simeq A_{(i, i)} \simeq h^{*} h_{!} A_{(i, i)}=h_{!} A_{(i, i)}
$$




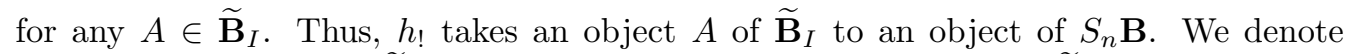
the restriction of $h_{!}$to $\widetilde{\mathbf{B}}_{I}$ by the same letter. To show that $h_{!}: \widetilde{\mathbf{B}}_{I} \longrightarrow S_{n} \mathbf{B}$ is an equivalence, we must check that the adjunction morphism

$$
h_{!} h^{*} B \longrightarrow B
$$

is an isomorphism for any $B \in S_{n} \mathbf{B}$. By the isomorphism axiom, it suffices to prove that this map is an isomorphism at each point $(i, j) \in \operatorname{Ar} \Delta^{n}$. Obviously, it is so at each $(i, j) \in I \cup(0,0)$.

Given $1 \leq i<j \leq n$, consider the square $a_{0, i, j}: \square \longrightarrow$ Ar $\Delta^{n}$. We denote by $\alpha$ the restriction of $a_{0, i, j}$ to $\ulcorner$. The morphism (6) induces a morphism

$$
\alpha^{*} h_{!} h^{*} B \longrightarrow \alpha^{*} B
$$

and also a morphism

$$
h_{!} h^{*} B_{(i, j)} \simeq \underline{\operatorname{Holim}}_{\ulcorner} \alpha^{*} h_{!} h^{*} B \longrightarrow \underline{\operatorname{Holim}}_{\ulcorner} \alpha^{*} B \simeq B_{(i, j)} .
$$

Here we have used Lemmas 1.7 and 2.3. We see that the latter morphism is an isomorphism whenever so is (7). Since $\operatorname{Im} \alpha \subset I$, it follows that (7) is always an isomorphism. Thus, (6) is an isomorphism at each $(i, j) \in \operatorname{Ar} \Delta^{n}$; hence, $h_{!}$and $h^{*}$ are mutual inverses by the isomorphism axiom.

Since $\ell=h g$, the functor $\ell^{*}=g^{*} h^{*}: S_{n} \mathbf{B} \longrightarrow \mathbf{B}_{\Delta^{n-1}}$ is an equivalence because so are both $g^{*}$ and $h^{*}$ (see above).

Remark. Observe that if $\mathbf{B}$ is a left system of diagram categories or a left pointed dérivateur, then a quasiinverse to $\ell^{*}$ is given by $h_{!} f^{*}\left(h_{!} g_{*}\right)$.

We denote by $\mathbf{B}(I)$ the left system of diagram categories or the left pointed dérivateur defined as $\mathbf{B}(I)_{J}=\mathbf{B}_{I \times J}$. Every map $f: I \longrightarrow J$ yields a functor $f^{*}: \mathbf{B}(J) \longrightarrow \mathbf{B}(I)$. Below we shall need the following statement.

Proposition 3.2. The structure functor $f^{*}: \mathbf{B}(J)_{0}=\mathbf{B}_{J} \longrightarrow \mathbf{B}(I)_{0}=\mathbf{B}_{I}$ respects the finite products and coproducts.

Proof. The proof is similar to that in [5, Subsection 1.4.7].

By Propositions 1.8 and 2.4, $f^{*}: \mathbf{B}(J) \longrightarrow \mathbf{B}(I)$ preserves the co-Cartesian squares. Therefore, we obtain a functor

$$
f^{*}: S_{n} \mathbf{B}(J) \longrightarrow S_{n} \mathbf{B}(I)
$$

(we denote it by the same letter), and for any bimorphism $\varphi: f \longrightarrow g$, the bimorphism $\varphi^{*}$ induces a natural transformation of functors

$$
S_{n} \mathbf{B}(J) \stackrel{f^{*}}{\longrightarrow} S_{g^{*}} \mathbf{B}(I) .
$$

We put $\mathbf{S}_{n} \mathbf{B}_{I}=S_{n} \mathbf{B}(I)$. Then $\mathbf{S}_{n} \mathbf{B}$ is a presystem of diagram categories or a prédérivateur, respectively. $\mathbf{S}_{0} \mathbf{B}$ is trivial, and for $n \geq 1$ Proposition 3.1 implies an equivalence

$$
\mathbf{S}_{n} \mathbf{B} \simeq \mathbf{B}\left(\Delta^{n-1}\right)
$$

Since $\mathbf{B}\left(\Delta^{n-1}\right)$ is a left system of diagram categories or a left pointed dérivateur, so is $\mathbf{S}_{n} \mathbf{B}$. Thus, we obtain a simplicial left system of diagram categories (respectively, a left pointed dérivateur)

$$
\text { S.B : } \Delta^{n} \longmapsto \mathbf{S}_{n} \mathbf{B} \text {. }
$$

Consider the following simplicial category:

$$
S . \mathbf{B}: \Delta^{n} \longmapsto S_{n} \mathbf{B} \text {. }
$$


For any $n \geq 0$, let $i S_{n} \mathbf{B}$ denote the subcategory of $S_{n} \mathbf{B}$ whose objects are those of $S_{n} \mathbf{B}$ and whose morphisms are isomorphisms in $S_{n} \mathbf{B}$, and let $i . S_{n} \mathbf{B}$ be the nerve of $i S_{n} \mathbf{B}$. We arrive at the following bisimplicial object:

$$
\text { i.S. : } \Delta^{m} \times \Delta^{n} \mapsto i_{m} S_{n} \mathbf{B} .
$$

Lemma 3.3. The space $|i . S . \mathbf{B}|$ is connected.

Proof. A geometric realization of a bisimplicial set is the diagonal. If $O_{1}, O_{2} \in i_{0} S_{0} \mathbf{B}$ and $f: O_{1} \longrightarrow O_{2}$ is a unique arrow in $S_{0} \mathbf{B}$ connecting $O_{1}$ and $O_{2}$, then for $A=\sigma_{0}(f) \in$ $i_{1} S_{1} \mathbf{B}$ we have $\partial_{0} A=O_{2}, \partial_{1} A=O_{1}$.

Definition. The Grothendieck group $K_{0}(\mathbf{B})$ is the group generated by the set of isomorphism classes $[B]$ of objects of $\mathbf{B}_{0}$ with the relations $[B]=[A] \cdot[C]$ for every $E \in S_{2} \mathbf{B}$ such that $E_{(0,1)}=A, E_{(0,2)}=B$, and $E_{(1,2)}=C$.

Lemma 3.4. $\pi_{1}|i . S . \mathbf{B}| \simeq K_{0}(\mathbf{B})$.

Proof. $\pi_{1}|i . S . \mathbf{B}|$ is the free group on $\pi_{0}\left|i . S_{1} \mathbf{B}\right|$ modulo the relations $d_{1}(x)=d_{2}(x) d_{0}(x)$ for every $x \in \pi_{0}\left|i . S_{2} \mathbf{B}\right|$. This follows from the relation $\pi_{1}\left|i . S_{0} \mathbf{B}\right|=0$ and the BousfieldFriedlander homotopy spectral sequence [17]. The set $\pi_{0}\left|i . S_{1} \mathbf{B}\right|$ is the set of isomorphism classes of objects in $\mathbf{B}_{0}, \pi_{0}\left|i . S_{2} \mathbf{B}\right|$ is the set of isomorphism classes of objects in $S_{2} \mathbf{B}$, and the maps $d_{i}: S_{2} \mathbf{B} \longrightarrow S_{1} \mathbf{B}$ send $E$ to $E_{(1,2)}, E_{(0,2)}$, and $E_{(0,1)}$, respectively.

Let $\mathcal{A}$ be an exact category. Its bounded derived category $D^{b}(\mathcal{A})$ is constructed as follows (here we use Keller's definitions [18]).

Let $H^{b}(\mathcal{A})$ be the homotopy category of the category of bounded complexes $\mathcal{C}=$ $C^{b}(\mathcal{A})$, i.e., the quotient category of $\mathcal{C}$ modulo homotopy equivalence. Let $A c(\mathcal{A})$ denote the full subcategory of $H^{b}(\mathcal{A})$ consisting of acyclic complexes. A complex

$$
X^{n} \longrightarrow X^{n+1} \longrightarrow X^{n+2}
$$

is said to be acyclic if each map $X^{n} \longrightarrow X^{n+1}$ decomposes in $\mathcal{A}$ as $X^{n} \stackrel{e_{n}}{\rightarrow} D^{n} \stackrel{m_{n}}{\longmapsto} X^{n+1}$, where $e_{n}$ is an (admissible) epimorphism and $m_{n}$ is an (admissible) monomorphism; furthermore, $D^{n} \stackrel{m_{n}}{\longmapsto} X^{n+1} \stackrel{e_{n+1}}{\rightarrow} D^{n+1}$ must be an exact sequence.

If an exact category is idempotent complete, then every contractible complex is acyclic. Denote by $\mathcal{N}=\mathcal{N}_{\mathcal{A}}$ the full subcategory of $H^{b}(\mathcal{A})$ whose objects are the complexes isomorphic in $H^{b}(\mathcal{A})$ to acyclic complexes. There is another description of $\mathcal{N}$. Let $\mathcal{A} \longrightarrow \tilde{\mathcal{A}}$ be the universal additive functor to an idempotent complete exact category $\tilde{\mathcal{A}}$. It is exact and respects exactness, and $\mathcal{A}$ is closed under extensions in $\tilde{\mathcal{A}}$ (see [19, A.9.1]). Then a complex with entries in $\mathcal{A}$ belongs to $\mathcal{N}$ if and only if its image in $H^{b}(\tilde{\mathcal{A}})$ is acyclic. The category $\mathcal{N}_{\tilde{\mathcal{A}}}=A c(\tilde{\mathcal{A}})$ is a thick subcategory in $H^{b}(\tilde{\mathcal{A}})$. Note that a complex over $\tilde{\mathcal{A}}$ is acyclic if and only if it has trivial homology computed in an ambient Abelian category. It follows that $\mathcal{N}$ is a thick subcategory in $H^{b}(\mathcal{A})$. Denote by $\Sigma$ the multiplicative system associated with $\mathcal{N}$; we call the elements of $\Sigma$ quasiisomorphisms. A morphism $s$ is a quasiisomorphism if and only if in any triangle

$$
L \stackrel{s}{\longrightarrow} M \longrightarrow N \longrightarrow L[1]
$$

the complex $N$ belongs to $\mathcal{N}$.

The derived category is defined as follows:

$$
D^{b}(\mathcal{A})=H^{b}(\mathcal{A}) / \mathcal{N}=H^{b}(\mathcal{A})\left[\Sigma^{-1}\right] .
$$

Clearly, a map is a quasiisomorphism if and only if its image in $C^{b}(\tilde{\mathcal{A}})$ is a quasiisomorphism and if and only if its image in $D^{b}(\mathcal{A})$ is an isomorphism. 
Recall that the Grothendieck group $K_{0}\left(D^{b}(\mathcal{A})\right)$ is defined as the group generated by the set of isomorphism classes $[B]$ of objects of $D^{b}(\mathcal{A})$ with the relations $[B]=[A]+[C]$ for every triangle $A \longrightarrow B \longrightarrow C \longrightarrow A[1]$.

In accordance with [18] (consult also [16]), the hyperfunctor $I \longmapsto D^{b}\left(\mathcal{A}^{I}\right)$ yields a pointed bidérivateur with domain Dirf. It will be denoted by $\mathbf{D}^{b}(\mathcal{A})$.

Lemma 3.5. $K_{0}\left(\mathbf{D}^{b}(\mathcal{A})\right)=K_{0}\left(D^{b}(\mathcal{A})\right)$.

Proof. It suffices to observe that there is a one-to-one correspondence between the isomorphism classes of objects in $S_{2} \mathbf{D}^{b}(\mathcal{A})$ and the isomorphism classes of triangles in $D^{b}(\mathcal{A})$ (see $[16,6]$ for the details).

Definition. The algebraic $K$-theory for a small left system of diagram categories with domain $\mathcal{O r d}^{\star}$ or for a left pointed dérivateur $\mathbf{B}$ with domain $\mathcal{D}$ ia is given by the pointed space

$$
K(\mathbf{B})=\Omega|i . S . \mathbf{B}|
$$

(a fixed zero object 0 of $\mathbf{B}_{0}$ is taken as a basepoint). The $K$-groups of $\mathbf{B}$ are the homotopy groups of $K(\mathbf{B})$ :

$$
K_{*}(\mathbf{B})=\pi_{*}(\Omega|i . S . \mathbf{B}|)=\pi_{*+1}(|i . S . \mathbf{B}|) .
$$

Convention. We shall also denote by $0 \in \mathbf{B}_{I}$ the object const ${ }^{*}$, where const $: I \longrightarrow 0$ is the constant map and 0 is the fixed zero object of $\mathbf{B}_{0}$. Let (L.s.d.c., Left pointed dérivateurs) denote the corresponding categories of left systems of diagram categories and left pointed dérivateurs and right exact functors. In order to make the map

$$
\text { (L.s.d.c., Left pointed dérivateurs) } \stackrel{K}{\longrightarrow} \text { (Spaces) }
$$

functorial, in what follows we assume that $\iota_{F, f}: f^{*} F \longrightarrow F f^{*}$ are identities for any right exact morphism $F: \mathbf{A} \longrightarrow \mathbf{B}$ and any map $f$ in $\mathcal{D}$ ia.

Any right exact morphism $F: \mathbf{A} \longrightarrow \mathbf{B}$ induces a map $F_{*}: K(\mathbf{A}) \longrightarrow K(\mathbf{B})$ of spaces and of their homotopy groups $K_{i}(\mathbf{A}) \longrightarrow K_{i}(\mathbf{B})$.

We can apply the $S$-construction to each $\mathbf{S}_{n} \mathbf{B}$, obtaining a bisimplicial left system of diagram categories or a bisimplicial left pointed dérivateur, respectively. Iterating this construction, we can form the multisimplicial object $\mathbf{S} .{ }^{n} \mathbf{B}=$ S.S. $\cdots \mathbf{S} . \mathbf{B}$ and the multisimplicial categories $i S .{ }^{n} \mathbf{B}$ of isomorphisms. Assuming the additivity theorem, we show that $\left|i . S .{ }^{n} \mathbf{B}\right|$ is the loop space of $\left|i . S .{ }^{n+1} \mathbf{B}\right|$ for any $n \geq 1$ and that the sequence

$$
\Omega|i . S . \mathbf{B}|, \quad \Omega|i . S . S . \mathbf{B}|, \quad \ldots, \quad \Omega\left|i . S .{ }^{n} \mathbf{B}\right|, \quad \ldots
$$

forms a connected $\Omega$-spectrum KB. In this case, the $K$-theory of $\mathbf{B}$ can be thought of in terms of this spectrum. This does not affect the $K$-groups, because

$$
\pi_{i}(\mathbf{K B})=\pi_{i}(K(\mathbf{B}))=K_{i}(\mathbf{B}), \quad i \geq 0 .
$$

The additivity theorem remains open for $K(\mathbf{B})$. Nevertheless, if the definition of $K$ theory as $\Omega|i . S . \mathbf{B}|$ is substituted with the infinite loop space

$$
\Omega^{\infty}\left|i . S .{ }^{\infty} \mathbf{B}\right|=\lim _{n} \Omega^{n}\left|i . S .^{n} \mathbf{B}\right|,
$$

then the additivity theorem holds true (excluding pathological cases we never have in practice).

Maltsiniotis [7] used the $Q$.-construction to define a $K$-theory space of a triangulated dérivateur. This construction can be extended to arbitrary left systems of diagram categories or left pointed dérivateurs if we replace bi-Cartesian squares in Maltsiniotis' definition by co-Cartesian squares. To be more precise, it is given by the bisimplicial category $Q \mathbf{B}=\left\{Q_{m, n} \mathbf{B}\right\}_{m, n \geq 0}$ with $Q_{m, n} \mathbf{B}$ being the full subcategory in $\mathbf{B}_{\Delta^{m}} \times \Delta^{n}$ such that every $X \in Q_{m, n} \mathbf{B}$ makes any square $i: \square \longrightarrow \Delta^{m} \times \Delta^{n}$ co-Cartesian. Let $i Q \mathbf{B}$ 
denote the corresponding maximal groupoid. Then $i . Q \mathbf{B}$ is a trisimplicial object, and the $K$-theory space is defined as $\Omega|\operatorname{diag}(i . Q \mathbf{B})|$.

In accordance with [20], the resulting $K$-theory is equivalent to that defined above in terms of the $S$-construction. The proof is based on [9, p. 334] and can be carried over to our setting without any problem.

\section{$\S 4$. Simplicial PREliminaries}

Multisimplicial sets will arise naturally in this work. It will be important for us to be able to handle them directly, without diagonalizing away all the structure. This direct procedure depends on a couple of lemmas, which we give below. We formulate them for bisimplicial sets, because the corresponding lemmas for multisimplicial sets can be obtained as immediate consequences, by taking suitable diagonals.

Lemma 4.1 ([8]). Let $X . . \longrightarrow Y$.. be a map of bisimplicial sets. Suppose that the map $X_{\cdot n} \longrightarrow Y_{\cdot n}$ is a homotopy equivalence for every $n$. Then $X . . \longrightarrow Y$.. is a homotopy equivalence.

Lemma 4.2 ([21, Subsection 5.2]). Let $X . . \longrightarrow Y . . \longrightarrow Z$.. be a sequence of bisimplicial sets such that $X . . \longrightarrow Z$.. is constant. Suppose that $X_{\cdot_{n}} \longrightarrow Y_{{ }_{n}} \longrightarrow Z_{\cdot_{n}}$ is a fibration up to homotopy for every $n$. Suppose further that $Z_{\cdot n}$ is connected for every $n$. Then $X . . \longrightarrow Y . . \longrightarrow Z$.. is a fibration up to homotopy.

Lemma 4.3. Let $\mathcal{A}$ and $\mathcal{B}$ be two small simplicial categories such that the underlying sets of objects form simplicial sets, and let $i \mathcal{A}$ and $i \mathcal{B}$ denote the corresponding simplicial subcategories of isomorphisms. Then every equivalence $F: \mathcal{A} \longrightarrow \mathcal{B}$ induces a homotopy equivalence of bisimplicial objects $F: i . \mathcal{A} \longrightarrow i . \mathcal{B}$. In particular, if $\mathcal{A}$ and $\mathcal{B}$ happen to be two left systems of diagram categories or two left pointed dérivateurs, then every right exact equivalence $F: \mathcal{A} \longrightarrow \mathcal{B}$ induces a homotopy equivalence $F: i . S . \mathcal{A} \longrightarrow$ i.S.B.

Proof. Straightforward.

Let $C$ and $D$ be two simplicial objects in a category $\mathcal{C}$ and let $\Delta / \Delta^{1}$ denote the category of objects over $\Delta^{1}$ in $\Delta$; the objects are the morphisms $\Delta^{n} \longrightarrow \Delta^{1}$. For any simplicial object $C$ in $\mathcal{C}$, let $C^{*}$ denote the composition

$$
\begin{gathered}
\left(\Delta / \Delta^{1}\right)^{\mathrm{op}} \longrightarrow \Delta^{\mathrm{op}} \stackrel{C}{\longrightarrow} \mathcal{C}, \\
\left(\Delta^{n} \longrightarrow \Delta^{1}\right) \longmapsto \Delta^{n} \longmapsto C_{n} .
\end{gathered}
$$

Then a simplicial homotopy of maps from $C$ to $D$ is a natural transformation $C^{*} \longrightarrow D^{*}$; see $[9$, p. 335].

There is a functor $P: \Delta \longrightarrow \Delta$ with $P \Delta^{n}=\Delta^{n+1}$ such that the natural map $s_{0}: \Delta^{n} \longrightarrow \Delta^{n+1}=P \Delta^{n}$ is a natural transformation $\operatorname{id}_{\Delta} \longrightarrow P$. This map is obtained by formally adding an initial object $0^{\prime}$ to each $\Delta^{n}$ and then identifying $\left\{0^{\prime}<1<\cdots<n\right\}$ with $\Delta^{n+1}$. Thus, $P\left(s_{i}\right)=s_{i+1}$ and $P\left(d_{i}\right)=d_{i+1}$. If $A$ is a simplicial object in $\mathcal{A}$, the path space $P A$ is the simplicial object obtained by composing $A$ with $P$. Therefore, $P A_{n}=A_{n+1}$, and the $i$ th face operator on $P A$ is the $\partial_{i+1}$ of $A$, and the $i$ th degeneracy operator on $P A$ is the $\sigma_{i+1}$ of $A$. Moreover, the maps $\partial_{0}: A_{n+1} \longrightarrow A_{n}$ form a simplicial $\operatorname{map} P A \longrightarrow A$.

We write $A_{0}$ for the constant simplicial object at $A_{0}$. The natural maps $\sigma_{0}^{n+1}: A_{0} \longrightarrow$ $A_{n+1}$ form a simplicial map $\iota: A_{0} \longrightarrow P A$, and the maps $A_{n+1} \longrightarrow A_{0}$ induced by the canonical inclusion of $\Delta^{0}=\{0\}$ in $\Delta^{n+1}$ form a simplicial map $\rho: P A \longrightarrow A_{0}$ such that $\rho \iota$ is the identity on $A_{0}$. It is well known that $\iota \rho$ is homotopic to the identity on $P A$. Therefore, $P A$ is homotopy equivalent to $A_{0}$. 


\section{$\S 5 . \Gamma$-SPACES}

In this section we use Segal's machine [8] to get some information about the $K$-theory $K(\mathbf{B})$. We start with preparations.

Given a finite set $T$, we denote by $\mathcal{P}(T)$ the set of subsets of $T$; the set $\{1,2, \ldots, n\}$ is denoted by $\mathbf{n}$.

Definition. I. $\Gamma$ is the category whose objects are all finite sets, and whose morphisms from $S$ to $T$ are the maps $\theta: S \longrightarrow \mathcal{P}(T)$ such that $\theta(\alpha)$ and $\theta(\beta)$ are disjoint whenever $\alpha \neq \beta$. The composition of $\theta: S \longrightarrow \mathcal{P}(T)$ and $\varphi: T \longrightarrow \mathcal{P}(U)$ is $\psi: S \longrightarrow \mathcal{P}(U)$, where $\psi(\alpha)=\bigcup_{\beta \in \theta(\alpha)} \varphi(\beta)$.

II. A $\Gamma$-space is a contravariant functor $A$ from $\Gamma$ to spaces such that

(a) $A(\mathbf{0})$ is contractible, and

(b) for any $n$ the map $p_{n}: A(\mathbf{n}) \longrightarrow A(\mathbf{1}) \times \cdots \times A(\mathbf{1})$ induced by the maps $i_{k}: \mathbf{1} \longrightarrow$ $\mathbf{n}$ in $\Gamma$, where $i_{k}(1)=\{k\} \subset \mathbf{n}$, is a homotopy equivalence.

We shall call $A(\mathbf{1})$ the underlying space.

There is a covariant functor $\Delta \longrightarrow \Gamma$ that takes $\Delta^{m}$ to $\mathbf{m}$ and $f: \Delta^{m} \longrightarrow \Delta^{n}$ to $\theta(i)=\{j \in \mathbf{n} \mid f(i-1)<j \leq f(i)\}$. Using this functor, we can regard $\Gamma$-spaces as simplicial spaces.

The realization functor $A \longrightarrow|A|$ used by Segal for simplicial spaces is slightly different from the usual one (see $[8$, Appendix A]). If $A$ is a $\Gamma$-space, its realization will mean the realization of the simplicial space it determines.

Definition. If $A$ is a $\Gamma$-space, its classifying space is the $\Gamma$-space $B A$ such that, for any finite set $S, B A(S)$ is the realization of the $\Gamma$-space $T \longmapsto A(S \times T)$.

If $A$ is a $\Gamma$-space, then the spaces $A(\mathbf{1}), B A(\mathbf{1}), B^{2} A(\mathbf{1}), \ldots$ form a spectrum, denoted by $\mathbf{B} A$. The reason of introducing $\Gamma$-spaces is that they arise naturally from categories.

Definition. A $\Gamma$-category is a contravariant functor $\mathcal{C}$ from $\Gamma$ to categories such that

(a) $\mathcal{C}(\mathbf{0})$ is equivalent to the category with one object and one morphism;

(b) for each $n$, the functor $p_{n}: \mathcal{C}(\mathbf{n}) \longrightarrow \mathcal{C}(\mathbf{1}) \times \cdots \times \mathcal{C}(\mathbf{1})$ induced by the maps $i_{k}: \mathbf{1} \longrightarrow \mathbf{n}$ in $\Gamma$ is an equivalence of categories.

If $\mathcal{C}$ is a $\Gamma$-category, $|\mathcal{C}|$ is a $\Gamma$-space. Here $|\mathcal{C}|$ means the functor $S \longmapsto|\mathcal{C}(S)|$.

$\Gamma$-categories arise in the following way. Let $\mathcal{C}$ be a category with sums and with a zero object 0 . If $S$ is a finite set, let $\mathcal{P}(S)$ denote the category of subsets of $S$ and their inclusions - this should not cause confusion with the earlier use of $\mathcal{P}(S)$. Let $\mathcal{C}(S)$ denote the category whose objects are the functors from $\mathcal{P}(S)$ that take disjoint unions to sums. The morphisms of $\Gamma$ were defined in such a way that the morphisms from $S$ to $T$ in $\Gamma$ correspond precisely to the functors from $\mathcal{P}(S)$ to $\mathcal{P}(T)$ that preserve disjoint unions. Then the category $i \mathcal{C}(S)$ of isomorphisms in $\mathcal{C}(S)$ satisfies the above definition.

Conforming to the terminology and notation of [9, Subsection 1.8], we denote the resulting simplicial category by N.C. By definition, $N_{0} \mathcal{C}=0$ and $N_{n} \mathcal{C}=\mathcal{C}(\mathbf{n})$ for each $n \geq 1$. We refer to the simplicial category N.C as the nerve with respect to the composition law. By construction, the space $|i . N . \mathcal{C}|$ is $B|i . \mathcal{C}|(\mathbf{1})$. Let $N . \mathbf{B}$ denote the nerve with respect to the composition law associated with the category $\mathbf{B}_{0}$.

Observe that any functor $f: \mathcal{C} \longrightarrow \mathcal{D}$ respecting sums yields a map of bisimplicial objects

$$
f^{*}: i . N . \mathcal{C} \longrightarrow i . N . \mathcal{D} \text {. }
$$

It follows that, for a given left system of diagram categories or a left pointed dérivateur $\mathbf{B}$, we can also produce the multisimplicial categories $i N \cdot{ }^{m} S .{ }^{n} \mathbf{B}, m, n \geq 0$, and the spaces $\left|i . N .{ }^{m} S .{ }^{n} \mathbf{B}\right|$ by iterating the $N$.- and $S$.-constructions. 
Proposition 5.1. $|i . S . \mathbf{B}|$ is canonically an infinite loop space, and hence so is the $K$ theory space $K(\mathbf{B})$.

Proof. The above considerations show that $|i . S . \mathbf{B}|$ is the underlying space of a $\Gamma$-space, with respect to the composition law produced by the coproduct.

Let $F: \mathbf{A} \longrightarrow \mathbf{B}$ be a right exact functor between left systems of diagram categories or left pointed dérivateurs, respectively. Let $N_{n}(\mathbf{A} \longrightarrow \mathbf{B})$ denote the fiber product of the diagram

$$
N_{n} \mathbf{A} \stackrel{F}{\longrightarrow} N_{n} \mathbf{B} \stackrel{\partial_{0}}{\longleftarrow}(P N . \mathbf{B})_{n}=N_{n+1} \mathbf{B} .
$$

An object of $N_{n}(\mathbf{A} \longrightarrow \mathbf{B})$ is a triple $(A, c, B)$, where $A \in N_{n} \mathbf{A}, B \in N_{n+1} \mathbf{B}$, and $c: F(A) \longrightarrow \partial_{0}(B)$ is an isomorphism in $N_{n} \mathbf{B}$. We obtain a simplicial category

$$
N .(\mathbf{A} \longrightarrow \mathbf{B}): \Delta^{n} \mapsto N_{n}(\mathbf{A} \longrightarrow \mathbf{B}) .
$$

For every $n$, there is a functor

$$
g: \mathbf{B}_{0}=N_{1} \mathbf{B} \longrightarrow N_{n}(\mathbf{A} \longrightarrow \mathbf{B})
$$

defined by $B \longmapsto\left(0,1, v^{*} B\right)$, where $v: \Delta^{n+1} \longrightarrow \Delta^{1}, i \longmapsto 0$ if $i=0$ and $i \longmapsto 1$ otherwise.

Regarding $\mathbf{B}_{0}$ as a trivial simplicial category, we obtain a sequence

$$
\mathbf{B}_{0} \stackrel{g}{\longrightarrow} N .(\mathbf{A} \longrightarrow \mathbf{B}) \stackrel{p}{\longrightarrow} N . \mathbf{A},
$$

where $p$ is the projection. The latter sequence gives rise to the sequence

$$
i . S . \mathbf{B} \stackrel{g}{\longrightarrow} i . N . S .(\mathbf{A} \longrightarrow \mathbf{B}) \stackrel{p}{\longrightarrow} i . N . S . \mathbf{A},
$$

where $N . S .(\mathbf{A} \longrightarrow \mathbf{B})=N .(S . \mathbf{A} \longrightarrow S . \mathbf{B})$. Note that the space $|i . N . S . \mathbf{A}|$ is $B|i . S . \mathbf{A}|(\mathbf{1})$, where $B|i . S . \mathbf{A}|$ is the $\Gamma$-space associated with $|i . S . \mathbf{A}|$.

Lemma 5.2. The sequence (8) is a fibration up to homotopy.

Proof. By Lemma 4.2, it suffices to show that for every $n$ the sequence

$$
i . S . \mathbf{B} \longrightarrow i . N_{n} S .(\mathbf{A} \longrightarrow \mathbf{B}) \longrightarrow i . N_{n} S . \mathbf{A}
$$

is a fibration (because the base term

$$
i . N_{n} S . \mathbf{A}=i . \operatorname{Hom}(\mathcal{P}(\mathbf{n}), S . \mathbf{A}) \simeq(i . S . \mathbf{A})^{n}
$$

is connected for every $n$ by Lemma 3.3). We shall show that the sequence is the same, up to homotopy, as the trivial fibration sequence associated with the product i.S.B $\times$ $i . N_{n} S$.A.

Let $u: \Delta^{1} \longrightarrow \Delta^{n+1}$ be the map $0 ; 1 \longmapsto 0 ; 1$. Also, consider the maps $d_{0}: \Delta^{n} \longrightarrow$ $\Delta^{n+1}$ and $s_{0}: \Delta^{n+1} \longrightarrow \Delta^{n}$. For any $B \in N_{n+1} \mathbf{B}$, we construct the diagram

$$
B^{\prime}=v^{*} u^{*} B \stackrel{\varphi}{\longrightarrow} B \stackrel{\psi}{\longleftarrow} B^{\prime \prime}=s_{0}^{*} \partial_{0} B .
$$

For any subset $S$ of $[\mathbf{n}+\mathbf{1}]$ :

$$
B_{S}^{\prime}= \begin{cases}B_{1} & \text { if } 1 \in S, \\ 0 & \text { if } 1 \notin S\end{cases}
$$

and

$$
B_{S}^{\prime \prime}= \begin{cases}B_{S \backslash\{1\}} & \text { if } 1 \in S, \\ B_{S} & \text { if } 1 \notin S,\end{cases}
$$

whence the definitions of $\varphi$ and $\psi$ follow. Note that $B_{S}^{\prime} \stackrel{\varphi_{S}}{\longrightarrow} B_{S} \stackrel{\psi_{S}}{\longleftarrow} B_{S}^{\prime \prime}$ belongs to $N_{2} \mathbf{B}$. 
The functor $N_{n}(\mathbf{A} \longrightarrow \mathbf{B}) \longrightarrow N_{n} \mathbf{A} \times \mathbf{B}_{0},(A, c, B) \longmapsto\left(A, B_{\{1\}}\right)$, is an equivalence of categories. A quasiinverse is given by the functor

$$
(A, B) \longmapsto\left(A, 1, s_{0}^{*} F A \amalg v^{*} B\right) .
$$

Then the induced map $i . N_{n} S .(\mathbf{A} \longrightarrow \mathbf{B}) \longrightarrow i . N_{n} S . \mathbf{A} \times i . S . \mathbf{B}$ is a homotopy equivalence by Lemma 4.1 .

This homotopy equivalence fits into the following commutative diagram:

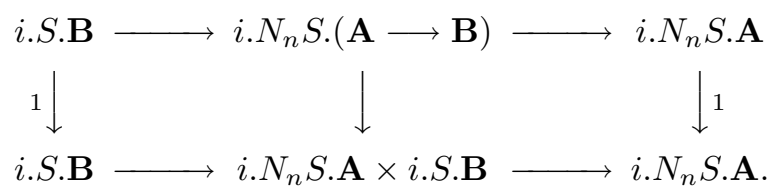

Being homotopy equivalent to the trivial fibration (the lower row of the diagram), the upper sequence is a fibration, as required.

As above, we can construct the sequence

$$
i . \mathbf{B}_{0} \longrightarrow P(i . N . \mathbf{B}) \longrightarrow i . N . \mathbf{B} .
$$

The composite map is constant and the middle term is contractible, so that we obtain a map that is well-defined up to homotopy,

$$
\left|i . \mathbf{B}_{0}\right| \longrightarrow \Omega|i . N . \mathbf{B}| \text {. }
$$

By naturality, in the above sequence we can replace $\mathbf{B}$ with the simplicial category $S . \mathbf{B}$. We obtain a sequence

$$
i . S . \mathbf{B} \longrightarrow P(i . N . S . \mathbf{B}) \longrightarrow i . N . S . \mathbf{B}
$$

where "P" refers to the $N$.-direction. By Lemma 5.2, the sequence is a fibration up to homotopy. Thus, $|i . S . \mathbf{B}| \longrightarrow \Omega|i . N . S . \mathbf{B}|$ is a homotopy equivalence. Therefore, more generally, by Lemma 4.1, the map $\left|i . N .{ }^{n} S . \mathbf{B}\right| \longrightarrow \Omega\left|i . N \cdot{ }^{n+1} S . \mathbf{B}\right|$ is also a homotopy equivalence. This results in the spectrum

$$
n \longmapsto\left|i . N .{ }^{n} S . \mathbf{B}\right| \text {, }
$$

which is actually an $\Omega$-spectrum. This is none other than the spectrum $n \longmapsto B^{n}|i . S . \mathbf{B}|(\mathbf{1})$ produced by Segal's machine.

Since all the maps in the sequence

$$
|i . S . \mathbf{B}| \longrightarrow \Omega|i . N . S . \mathbf{B}| \longrightarrow \Omega \Omega|i . N . N . S . \mathbf{B}| \longrightarrow \cdots
$$

are homotopy equivalences, so is the map

$$
|i . S . \mathbf{B}| \longrightarrow \Omega^{\infty}\left|i . N .{ }^{\infty} S . \mathbf{B}\right|=\lim _{n} \Omega^{n}\left|i . N .{ }^{n} S . \mathbf{B}\right| .
$$

Corollary 5.3. Let $\mathbf{A} \longrightarrow \mathbf{B} \longrightarrow \mathbf{C}$ be a sequence of right exact morphisms of left systems of diagram categories or left pointed dérivateurs, respectively. Then the square

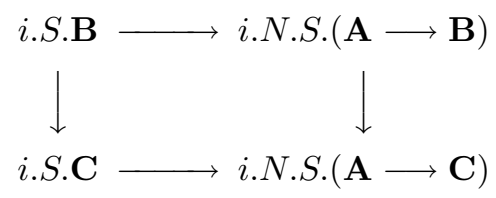

is homotopy Cartesian.

Proof. There is a commutative diagram

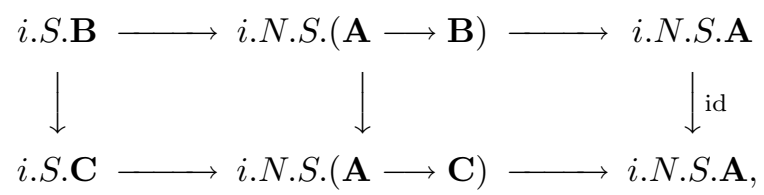


in which the rows are fibrations up to homotopy by Lemma 5.2. Therefore, the square on the left is homotopy Cartesian.

Corollary 5.4. 1) Any right exact morphism gives rise to a fibration

$$
i . S . \mathbf{B} \longrightarrow i . S . \mathbf{C} \longrightarrow i . N . S .(\mathbf{B} \longrightarrow \mathbf{C}) .
$$

2) If $\mathbf{C}$ is a retract of $\mathbf{B}$ (by right exact functors), then there is a splitting

$$
i . S . \mathbf{B} \simeq i . S . \mathbf{C} \times i . N . S .(\mathbf{C} \longrightarrow \mathbf{B}) .
$$

Proof. 1) If $\mathbf{A}=\mathbf{B}$, then the space $|i . N . S .(\mathbf{A}=\mathbf{A})|$ is contractible, whence the first claim.

2) Since the composition $\mathbf{A} \longrightarrow \mathbf{B} \longrightarrow \mathbf{C}$ is an identity map, i.N.S. $(\mathbf{A} \longrightarrow \mathbf{C})$ is contractible, and then 2) is implied by Corollary 5.3 .

\section{$\S 6$. The ADDitivity TheOREM}

Let $\mathbf{B}$ be either a left system of diagram categories or a left pointed dérivateur. Denote by $\mathbf{E}_{0}$ the full subcategory in $\mathbf{B}_{\square}$ consisting of the co-Cartesian squares $B \in \mathbf{B}_{\square}$ with $B_{(1,0)}$ isomorphic to zero. Replacing $\mathbf{B}$ by $\mathbf{B}(I)$, we define a category $\mathbf{E}_{I}$ similar to $\mathbf{E}_{0}$. This leads to a left system of diagram categories or a left pointed dérivateur $\mathbf{E}$, respectively.

Lemma 6.1. The map $l: \Delta^{1} \longrightarrow \square, i \longmapsto(0, i)$, induces an equivalence of categories $l^{*}: \mathbf{E}_{0} \longrightarrow \mathbf{B}_{\Delta^{1}}$ and also a right exact equivalence $\mathbf{E} \longrightarrow \mathbf{B}\left(\Delta^{1}\right)$.

Proof. The map $l$ factors as

$$
\Delta^{1} \stackrel{g}{\longrightarrow}\ulcorner\stackrel{h}{\longrightarrow} \square .
$$

The proof of Proposition 3.1 shows that $l^{*}: \mathbf{E}_{0} \longrightarrow \mathbf{B}_{\Delta^{1}}$ is an equivalence (in the case of left pointed dérivateurs, use the fact that $g$ is an open immersion). Obviously, the induced morphism $\mathbf{E} \longrightarrow \mathbf{B}\left(\Delta^{1}\right)$ is an equivalence. It is right exact by Propositions 1.8 and 2.4 .

Corollary 6.2. The map $f: \square \longrightarrow \operatorname{Ar} \Delta^{2},(i, j) \longmapsto(i, j+1)$, induces an equivalence of categories $f^{*}: S_{2} \mathbf{B} \longrightarrow \mathbf{E}_{0}$ and also a right exact equivalence $\mathbf{S}_{2} \mathbf{B} \longrightarrow \mathbf{E}$.

Proof. Let $\ell: \Delta^{1} \longrightarrow$ Ar $\Delta^{2}$ be the map $i \longmapsto(0, i+1)$. It factors as $\Delta^{1} \stackrel{l}{\longrightarrow} \stackrel{f}{\longrightarrow} \operatorname{Ar} \Delta^{2}$, where $l$ is the map of Lemma 6.1. By Proposition 3.1, it follows that $\ell^{*}=l^{*} f^{*}$ : $\mathbf{S}_{2} \mathbf{B} \longrightarrow \mathbf{B}_{\Delta^{1}}$ is an equivalence. By Lemma $6.1, l^{*}$ is an equivalence, whence $f^{*}$ is also an equivalence.

Lemma 6.3. Let $\mathbf{B}$ be either a left system of diagram categories or a left pointed dérivateur, and let $B \in \mathbf{B}_{\square}$ be a co-Cartesian square such that the map $B_{(0,0)} \longrightarrow B_{(0,1)}$ (respectively, the map $\left.B_{(0,0)} \longrightarrow B_{(1,0)}\right)$ is an isomorphism. Then $B_{(1,0)} \longrightarrow B_{(1,1)}(r e-$ spectively, $\left.B_{(0,1)} \longrightarrow B_{(1,1)}\right)$ is also an isomorphism. On the other hand, a square in which two parallel arrows are isomorphisms is co-Cartesian.

Proof. Suppose that the map $B_{(0,0)} \longrightarrow B_{(0,1)}$ is an isomorphism. Let $q: \square \longrightarrow \Delta^{1}$ denote the functor $(\varepsilon, \eta) \longmapsto \varepsilon$, and let $i: \Delta^{1} \longrightarrow \square$ be the functor $\varkappa \longmapsto(\varkappa, 0)$. Then $i$ is a left adjoint to $q$, whence $i_{!} \simeq q^{*}$. The map $i$ factors as

$$
\Delta^{1} \stackrel{l}{\longrightarrow}\left\ulcorner\stackrel{i_{\ulcorner}}{\longrightarrow} \square,\right.
$$

where $l(\varkappa)=(\varkappa, 0)$. By Propositions 1.9 and 2.5 , the object $i_{!} i^{*} B \simeq i_{r !}\left(l_{!} i^{*} B\right)$ is coCartesian. 
Let $\beta:\left(i_{i} i^{*} B \simeq\right) q^{*} i^{*} B \longrightarrow B$ be the adjunction morphism. Then $\beta_{(0,0)}=\beta_{(1,0)}=1$, and $\beta_{(0,1)}$ is an isomorphism by assumption. It follows that $i_{\ulcorner}^{*} \beta$ is an isomorphism. Consider the commutative square

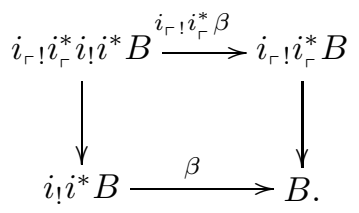

The upper arrow is an isomorphism. The vertical maps are isomorphisms, because both $B$ and $i_{!} i^{*} B$ are co-Cartesian. We see that $\beta$ is also an isomorphism. This implies that $B_{(1,0)} \longrightarrow B_{(1,1)} \simeq \beta_{(1,1)}$ is an isomorphism. The corresponding assertion in the case where $B_{(0,0)} \longrightarrow B_{(1,0)}$ is an isomorphism is deduced from the first assertion by application of the autoequivalence $\tau: \square \longrightarrow \square$ transposing the vertices $(1,0)$ and $(0,1)$.

On the other hand, if both $B_{(0,0)} \longrightarrow B_{(0,1)}$ and $B_{(1,0)} \longrightarrow B_{(1,1)}$ are isomorphisms, then $\beta: i_{!} i^{*} B \longrightarrow B$ is an isomorphism. Since $i_{i} i^{*} B$ is co-Cartesian, it follows that $B$ is also co-Cartesian.

We want to construct a functor $\alpha: \mathbf{B}_{0} \longrightarrow \mathbf{E}_{0}$ that takes an object $A \in \mathbf{B}_{0}$ to an object in $\mathbf{E}_{0}$ and which is depicted in $\mathbf{B}_{0}$ as the square

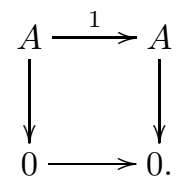

Let $s_{0}: \Delta^{1} \longrightarrow 0$ be a unique map. First, suppose that $\mathbf{B}$ is a left system of diagram categories and $d: \square^{\star} \longrightarrow \Delta^{1 \star}$ is the map $(0, i) \longmapsto i$ and $(1, i) \longmapsto \star$. Then we put $\alpha=d^{*} s_{0}^{*}$. If $\mathbf{B}$ is a left pointed dérivateur, we let $l: \Delta^{1} \longrightarrow \square$ be the map $i \longmapsto(0, i)$. Then $l$ is an open immersion, whence there is a right adjoint functor $l_{*}$ to $l^{*}$. In this case $\alpha=l_{*} s_{0}^{*}$.

Let $j: \square \longrightarrow \Delta^{1}$ be the map $(\varepsilon, \eta) \longmapsto \eta$. The morphism $j^{*}$ takes $B \in \mathbf{B}_{\Delta^{1}}$ to a square in $\mathbf{B}_{\square}$ which is depicted in $\mathbf{B}_{0}$ as the square

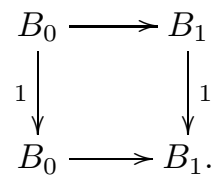

Let $\mathbf{B}$ be a left system of diagram categories, and let $u: \Delta^{1 \star} \longrightarrow 0^{\star}$ be the map $0 \longmapsto \star$ and $1 \longmapsto 0$. Then $u^{*}$ takes an object $B \in \mathbf{B}_{0}$ to one in $\mathbf{B}_{\Delta^{1}}$ with $u^{*} B_{0}=0$ and $u^{*} B_{1}=B$. We put $\beta=j^{*} u^{*}$. In its turn, if $\mathbf{B}$ is a left pointed dérivateur, consider the map $v: 0 \longrightarrow \Delta^{1}$ with $v(0)=1$. Then $v_{!} B_{0}=0$ and $v_{!} B_{1}=B$ for any $B \in \mathbf{B}_{0}$. In this case $\beta:=j^{*} v_{\text {! }}$.

The functor $\beta$ takes an object $B \in \mathbf{B}_{0}$ to the square

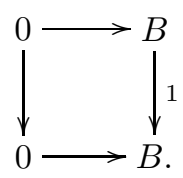

Let $\mathbf{B}$ be either a left system of diagram categories or a left pointed dérivateur, and let $\mathbf{B}^{\prime}$ and $\mathbf{B}^{\prime \prime}$ be either two left subsystems of diagram categories or two left pointed subdérivateurs such that the inclusion morphisms are right exact. There are three natural 
right exact morphisms $s, t, q: \mathbf{E} \longrightarrow \mathbf{B}$ taking an object $E \in \mathbf{E}_{\text {? }}$ to $E_{(0,0)}, E_{(0,1)}$, and $E_{(1,1)}$, respectively. We define $\mathbf{E}\left(\mathbf{B}^{\prime}, \mathbf{B}, \mathbf{B}^{\prime \prime}\right)$ as a presystem of diagram categories or as a prédérivateur that consists of the squares $E \in \mathbf{E}$ with $E_{(0,0)} \in \mathbf{B}^{\prime}$ and $E_{(1,1)} \in \mathbf{B}^{\prime \prime}$. Then $\mathbf{E}\left(\mathbf{B}^{\prime}, \mathbf{B}, \mathbf{B}^{\prime \prime}\right)$ is a left system of diagram categories or a left pointed dérivateur, respectively, because $\mathbf{E}\left(\mathbf{B}^{\prime}, \mathbf{B}, \mathbf{B}^{\prime \prime}\right)$ is equivalent to the fiber product of the diagram $\mathbf{E} \stackrel{(s, q)}{\longrightarrow} \mathbf{B} \times \mathbf{B} \longleftarrow \mathbf{B}^{\prime} \times \mathbf{B}^{\prime \prime}$. We note that $\mathbf{E}=\mathbf{E}(\mathbf{B}, \mathbf{B}, \mathbf{B})$

In order to obtain a unital and associative $H$-space structure for $|i . S . \mathbf{B}|$ induced by the coproduct $\amalg$ via the map

$$
|i . S . \mathbf{B}| \times|i . S . \mathbf{B}| \stackrel{\sim}{\longrightarrow}|i . S . \mathbf{B} \times i . S . \mathbf{B}| \stackrel{\amalg}{\longrightarrow}|i . S . \mathbf{B}|,
$$

we must have good choices for $A \amalg B, A, B \in S_{n} \mathbf{B}$, so that $f^{*}(A \amalg B)=f^{*}(A) \amalg f^{*}(B)$, where $f: \Delta^{m} \longrightarrow \Delta^{n}$ is a structure map in $\Delta$ (we always have an isomorphism between them because $f^{*}$ respects coproducts by Lemma 3.2 ). Then we would have a simplicial equivalence $\amalg(\amalg \times 1) \simeq \amalg(1 \times \amalg)$,

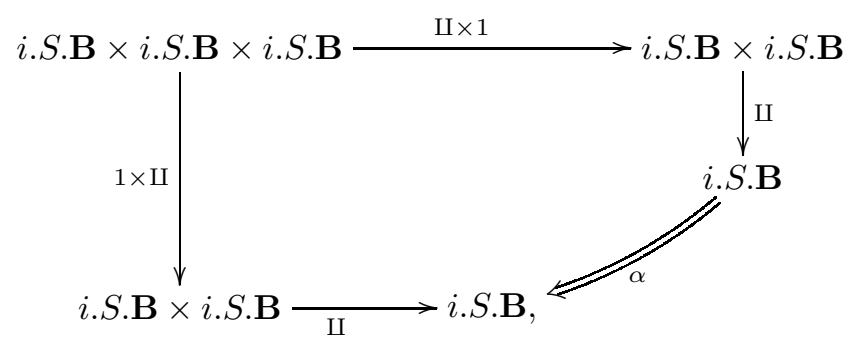

inducing a homotopy between them after realization. It would also follow that the two maps $i . S . \mathbf{B} \longrightarrow i . S . \mathbf{B}$ given by $B \longmapsto B \amalg 0$ and $B \longmapsto 0 \amalg B$ are homotopic to the identity map, implying that $|i . S . \mathbf{B}|$ is unital. It seems that we do not have enough data to produce such choices in general. We shall refer to this case as pathological. The latter term is caused by the observation that the required choices always exist in practice. Indeed, all left systems of diagram categories or left pointed dérivateurs arise in practice as the hyperfunctor $I \longmapsto \mathrm{HoC}^{I}$ with $\mathcal{C}$ closed under coproducts. Then the choices are made in $\mathcal{C}$.

Convention. In the rest of this section we assume $\mathbf{B}$ to be nonpathological.

By a right exact sequence $F^{\prime} \longrightarrow F \longrightarrow F^{\prime \prime}$ of right exact functors $\mathbf{B}^{\prime} \longrightarrow \mathbf{B}$ we mean a right exact functor $G: \mathbf{B}^{\prime} \longrightarrow \mathbf{E}=\mathbf{E}(\mathbf{B}, \mathbf{B}, \mathbf{B})$ such that $F^{\prime}=s \circ G, F=t \circ G$, and $F^{\prime \prime}=q \circ G$.

Proposition 6.4 (Equivalent formulations of the additivity theorem). Each of the following conditions implies the other three.

1) The projection

$$
i . S . \mathbf{E}\left(\mathbf{B}^{\prime}, \mathbf{B}, \mathbf{B}^{\prime \prime}\right) \longrightarrow i . S . \mathbf{B}^{\prime} \times i . S . \mathbf{B}^{\prime \prime}, \quad E \mapsto\left(E_{(0,0)}, E_{(1,1)}\right),
$$

is a homotopy equivalence.

2) The projection

$$
i . S . \mathbf{E} \longrightarrow i . S . \mathbf{B} \times i . S . \mathbf{B}, \quad E \mapsto\left(E_{(0,0)}, E_{(1,1)}\right),
$$

is a homotopy equivalence.

3) The following two maps are homotopic:

$i . S . \mathbf{E} \longrightarrow i . S . \mathbf{B}, \quad E \mapsto E_{(0,1)}$, and, respectively, $E \mapsto E_{(0,0)} \amalg E_{(1,1)}$. 
4) If $F^{\prime} \longrightarrow F \longrightarrow F^{\prime \prime}$ is a right exact sequence of right exact functors $\mathbf{B}^{\prime} \longrightarrow \mathbf{B}$, then there exists a homotopy

$$
|i . S . F| \simeq\left|i . S . F^{\prime}\right| \vee\left|i . S . F^{\prime \prime}\right| \text {. }
$$

Proof. 2) is a special case of 1), 3) is a special case of 4), and 4) follows from 3) by naturality.

So it will suffice to show the implications 2) $\Longrightarrow 3$ ) and 4) $\Longrightarrow 1$ ).

$2) \Longrightarrow 3)$. The desired homotopy $|i . S . t| \simeq|i . S .(s \vee q)|$ is obtained upon restriction along the map

$$
|i . S . \mathbf{B}| \times|i . S . \mathbf{B}| \longrightarrow|i . S . \mathbf{E}|, \quad(A, B) \mapsto \alpha A \amalg \beta B .
$$

Therefore, it suffices to know that this map is a homotopy equivalence. But this map is a section of the map in 2), so that it is a homotopy equivalence if so is the map in 2).

$4) \Longrightarrow 1$ ). First, we consider the maps $l: \Delta^{1} \longrightarrow \square, \varkappa \mapsto(\varkappa, 0)$, and $q: \square \longrightarrow \Delta^{1}$, $(\varepsilon, \eta) \mapsto \varepsilon$. Denote $\mathbf{E}_{?}^{\prime}=\left\{q^{*} l^{*} E \mid E \in \mathbf{E}\left(\mathbf{B}^{\prime}, \mathbf{B}, \mathbf{B}^{\prime \prime}\right)_{?}\right\}$. For $E \in \mathbf{E}\left(\mathbf{B}^{\prime}, \mathbf{B}, \mathbf{B}^{\prime \prime}\right)_{0}$, the object $q^{*} l^{*} E$ is depicted in $\mathbf{B}_{0}$ as

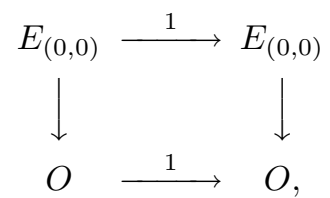

where $O=E_{(1,0)}$ is a zero object.

Also, let $i: \Delta^{1} \longrightarrow \square, \varkappa \mapsto(1, \varkappa)$, and $j: \square \longrightarrow \Delta^{1},(\varepsilon, \eta) \mapsto \eta$. Denote $\mathbf{E}_{?}^{\prime \prime}=$ $\left\{j^{*} i^{*} E \mid E \in \mathbf{E}\left(\mathbf{B}^{\prime}, \mathbf{B}, \mathbf{B}^{\prime \prime}\right)_{?}\right\}$. For $E \in \mathbf{E}\left(\mathbf{B}^{\prime}, \mathbf{B}, \mathbf{B}^{\prime \prime}\right)_{0}$, the object $j^{*} i^{*} E$ is depicted in $\mathbf{B}_{0}$ as

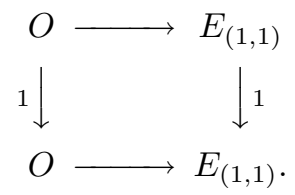

We shall construct a right exact morphism

$$
E \in \mathbf{E}\left(\mathbf{B}^{\prime}, \mathbf{B}, \mathbf{B}^{\prime \prime}\right) ? \mapsto E^{2} \in \mathbf{E}\left(\mathbf{E}^{\prime}, \mathbf{E}, \mathbf{E}^{\prime \prime}\right) ?
$$

such that $E^{2}$ is depicted in $\mathbf{E}_{0}$ as follows:

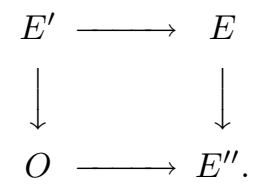

If we neglect the object at $(1,0) \in \square$, then the above diagram is depicted in $\mathbf{B}_{0}$ as

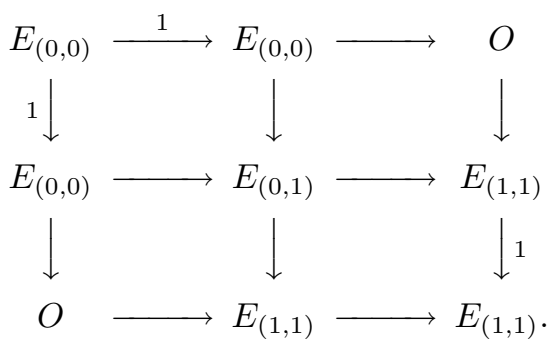

Then our assumption will imply immediately that

$$
i . S . \mathbf{E}\left(\mathbf{B}^{\prime}, \mathbf{B}, \mathbf{B}^{\prime \prime}\right) \longrightarrow i . S . \mathbf{E}^{\prime} \times i . S . \mathbf{E}^{\prime \prime}, \quad E \mapsto\left(q^{*} l^{*} E, j^{*} i^{*} E\right),
$$


is a homotopy equivalence with section $\left(E^{\prime}, E^{\prime \prime}\right) \mapsto E^{\prime} \amalg E^{\prime \prime}$. We construct the square $E^{2}$ in the following way. Consider two maps $\varphi, \psi: \square \longrightarrow \Delta^{1}$ defined by the rules

and

$$
(0,0),(0,1),(1,0) \stackrel{\varphi}{\mapsto} 0, \quad(1,1) \stackrel{\varphi}{\mapsto} 1,
$$

$$
(0,0) \stackrel{\psi}{\longmapsto} 0, \quad(0,1),(1,0),(1,1) \stackrel{\psi}{\longmapsto} 1 .
$$

For an object $A \in \mathbf{B}_{\Delta^{1}}$, the functors $\varphi^{*}$ and $\psi^{*}$ take $A$ to the squares that are depicted in $\mathbf{B}_{0}$ as

and
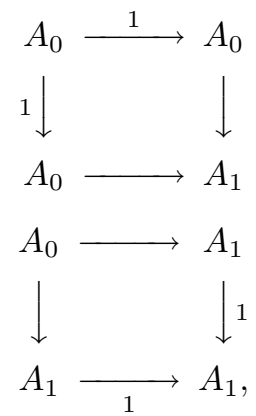

respectively.

An object $E \in \mathbf{E}\left(\mathbf{B}^{\prime}, \mathbf{B}, \mathbf{B}^{\prime \prime}\right)_{0}$ can be regarded as an object in $\mathbf{B}\left(\Delta^{1}\right)_{\Delta^{1}}$, and it is evaluated as $E_{(0,0)} \longrightarrow O$ at 0 and as $E_{(0,1)} \longrightarrow E_{(1,1)}$ at 1 . We embed $E$ in the coCartesian square $E^{1}=\left(1_{\Delta^{1}} \times \varphi\right)^{*} E$ in $\mathbf{B}\left(\Delta^{1}\right) \square$. After depicting $E^{1}$ in an appropriate way, we obtain the following diagram in $\mathbf{B}_{0}$ :

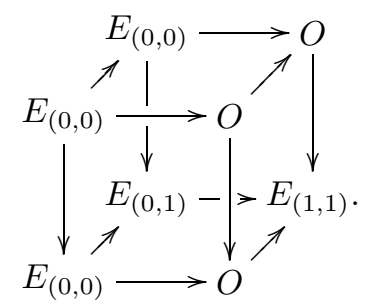

The object $E^{1}$ can be regarded as an object in $\mathbf{B}(\square)_{\Delta^{1}}$, and it is evaluated as the left square of the depicted cube at 0 and as the right square at 1 . We embed the $E^{1}$ in the coCartesian square $E^{2}=\left(\psi \times 1_{\square}\right)^{*} E^{1}$ in $\mathbf{B}(\square)_{\square}$. The construction of $E^{2}$ is completed. The morphism $E \in \mathbf{E}\left(\mathbf{B}^{\prime}, \mathbf{B}, \mathbf{B}^{\prime \prime}\right)_{?} \longmapsto E^{2} \in \mathbf{E}\left(\mathbf{E}^{\prime}, \mathbf{E}, \mathbf{E}^{\prime \prime}\right)_{\text {? }}$ is induced by $\left(\psi \times 1_{\square}\right)^{*}\left(1_{\Delta^{1}} \times \varphi\right)^{*}$.

It remains to show that the maps $f: E \in \mathbf{E}_{?}^{\prime} \longmapsto E_{(0,0)} \in \mathbf{B}_{\text {? }}$ and $g: E \in \mathbf{E}_{?}^{\prime \prime} \longmapsto$ $E_{(1,1)} \in \mathbf{B}_{\text {? }}$ induce a homotopy equivalence

$$
i . S . \mathbf{E}^{\prime} \times i . S . \mathbf{E}^{\prime \prime} \stackrel{(f, g)}{\longrightarrow} i . S . \mathbf{B}^{\prime} \times i . S . \mathbf{B}^{\prime \prime},
$$

because the map $p: i . S . \mathbf{E}\left(\mathbf{B}^{\prime}, \mathbf{B}, \mathbf{B}^{\prime \prime}\right) \longrightarrow i . S . \mathbf{B}^{\prime} \times i . S . \mathbf{B}^{\prime \prime}, E \longmapsto\left(E_{(0,0)}, E_{(1,1)}\right)$, is equal to the composition

$$
i . S . \mathbf{E}\left(\mathbf{B}^{\prime}, \mathbf{B}, \mathbf{B}^{\prime \prime}\right) \stackrel{\left(q^{*} l^{*}, j^{*} i^{*}\right)}{\longrightarrow} i . S . \mathbf{E}^{\prime} \times i . S . \mathbf{E}^{\prime \prime} \stackrel{(f, g)}{\longrightarrow} i . S . \mathbf{B}^{\prime} \times i . S . \mathbf{B}^{\prime \prime},
$$

and the left arrow is a homotopy equivalence by the above.

Let $\overline{\mathbf{B}}_{\text {? }}=\left\{X \in \mathbf{B}_{\Delta^{1} \times \text { ? }} \mid X_{1} \simeq 0\right\}$. Then $\overline{\mathbf{B}}$ and $\mathbf{E}^{\prime}$ are isomorphic because $l^{*} q^{*}=1_{\overline{\mathbf{B}}}$ and $\left.q^{*} l^{*} q^{*} l^{*}\right|_{\mathbf{E}^{\prime}}=1_{\mathbf{E}^{\prime}}$. In a similar way, let $\overline{\overline{\mathbf{B}}}_{\text {? }}=\left\{X \in \mathbf{B}_{\Delta^{1} \times}\right.$ ? $\left.\mid X_{0} \simeq 0\right\}$. Then $\overline{\overline{\mathbf{B}}}$ and $\mathbf{E}^{\prime \prime}$ are isomorphic because $i^{*} j^{*}=1_{\overline{\mathbf{B}}}$ and $\left.j^{*} i^{*} j^{*} i^{*}\right|_{\mathbf{E}^{\prime \prime}}=1_{\mathbf{E}^{\prime \prime}}$.

Finally, the proof of Proposition 3.1 shows that the morphism $\overline{\mathbf{B}} \longrightarrow \mathbf{B}^{\prime}$ induced by the map $0 \longmapsto 0 \in \Delta^{1}$ is an equivalence, as well as the morphism $\overline{\overline{\mathbf{B}}} \longrightarrow \mathbf{B}^{\prime \prime}$ induced by the map $0 \longmapsto 1 \in \Delta^{1}$. We are done. 
For a simplicial object $X$, let $P X \longrightarrow X$ be the projection induced by the face map $\partial_{0}: X_{n+1} \longrightarrow X_{n}$. If we regard $X_{1}$ as a trivial simplicial object, then there is an inclusion $X_{1} \longrightarrow P X$ resulting in a sequence $X_{1} \longrightarrow P X \longrightarrow X$.

In particular, we obtain a sequence $i . S_{1} \mathbf{B} \longrightarrow P(i . S . \mathbf{B}) \longrightarrow i . S . \mathbf{B}$. In view of the equivalence of $i . S_{1} \mathbf{B}$ with $i . \mathbf{B}_{0}$, this sequence can be rewritten as

$$
i . \mathbf{B}_{0} \stackrel{G}{\longrightarrow} P(i . S . \mathbf{B}) \stackrel{\partial_{0}}{\longrightarrow} i . S . \mathbf{B} .
$$

We show explicitly what the map $G$ is. Let $\ell^{*}: S_{1} \mathbf{B} \longrightarrow \mathbf{B}_{0}$ be the equivalence occurring in Proposition 3.1. A quasiinverse to $\ell^{*}$ is constructed as follows. Consider the open immersion $e: 0 \longmapsto 0 \in \Delta^{1}$. If $\mathbf{B}$ is a left system of diagram categories and $k: \Delta^{1} \longrightarrow 0$ is the morphism $0 ; 1 \mapsto 0 ; \star$, then $\left(k^{*} B\right)_{0}=B$ and $\left(k^{*} B\right)_{1}=0$ for every $B \in \mathbf{B}_{0}$. In its turn, if $\mathbf{B}$ is a left pointed dérivateur, then $\left(e_{*} B\right)_{0}=B$ and $\left(e_{*} B\right)_{1}=0$.

Next, let $p: \Delta^{1} \longrightarrow \operatorname{Ar} \Delta^{1}$ be the closed immersion $i \mapsto(i, 1)$, let $r: \operatorname{Ar} \Delta^{1 \star} \longrightarrow \Delta^{1 \star}$ be the morphism $(0,0) \mapsto \star,(0 ; 1,1) \mapsto 0 ; 1$, and let $B \in \mathbf{B}_{0}$. If $\mathbf{B}$ is a left system of diagram categories, then $\left(r^{*} k^{*} B\right)_{(0,0)}=\left(r^{*} k^{*} B\right)_{(1,1)}=0$ and $\left(r^{*} k^{*} B\right)_{(0,1)}=B$. Set $g=r^{*} k^{*}$. If $\mathbf{B}$ is a left pointed dérivateur, then $\left(p_{!} e_{*} B\right)_{(0,0)}=\left(p_{!} e_{*} B\right)_{(1,1)}=0$ and $\left(p_{!} e_{*} B\right)_{(0,1)}=B$. In this case $g:=p_{!} e_{*}$.

We consider the map $v: \Delta^{n+1} \longrightarrow \Delta^{1}, 0 \mapsto 0$ and $i \mapsto 1$ for $i \geq 1$. Set $G=$ $v^{*} g: \mathbf{B}_{0} \longrightarrow S_{n+1} \mathbf{B}$. Then the "values" of $G B$ at each $(i, j) \in \operatorname{Ar} \Delta^{n+1}$ look like this: $G B_{(i, j)}=0$ if $(i, j)=(0,0), i \geq 1$, and $G B_{(0, j)}=B$ for $j \geq 1$. Regarding $\mathbf{B}_{0}$ as a trivial simplicial category, we obtain the maps $G: \mathbf{B}_{0} \longrightarrow P S . \mathbf{B}$ and $G:\left|i . \mathbf{B}_{0}\right| \longrightarrow|P(i . S . \mathbf{B})|$.

The composition $\left|i . \mathbf{B}_{0}\right| \stackrel{G}{\longrightarrow}|P(i . S . \mathbf{B})| \longrightarrow|i . S . \mathbf{B}|$ is constant, and $|P(i . S . \mathbf{B})|$ is contractible (being homotopy equivalent to the contractible space $\left|i . S_{0} \mathbf{B}\right|$ ). Therefore, we obtain a map

well-defined up to homotopy.

$$
\left|i . \mathbf{B}_{0}\right| \longrightarrow \Omega|i . S . \mathbf{B}|,
$$

We present a couple of useful observations due to Waldhausen [9, p. 332].

Observation. The following two composite maps are homotopic:

$$
\left|i . \mathbf{E}_{0}\right| \underset{s \vee q}{\stackrel{t}{\longrightarrow}}\left|i . \mathbf{B}_{0}\right| \longrightarrow \Omega|i . S . \mathbf{B}| \text {. }
$$

Proof. This results from an inspection of $|i . S . \mathbf{B}|_{(2)}$, the 2-skeleton of $|i . S . \mathbf{B}|$, in the $S$.direction. We can identify $i \mathbf{B}_{0}$ with $i S_{1} \mathbf{B}$ and $i \mathbf{E}_{0}$ with $i S_{2} \mathbf{B}$.

The face maps from $i . S_{2} \mathbf{B}$ to $i . S_{1} \mathbf{B}$ correspond to the three maps $s, t, q$, respectively, and each of them can be seen from the diagram

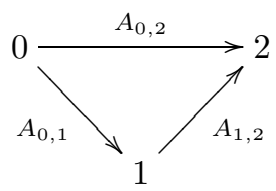

Consider the canonical map $\left|i . S_{2} \mathbf{B}\right| \times\left|\Delta^{2}\right| \longrightarrow|i . S . \mathbf{B}|_{(2)}$. Regarding the 2-simplex $\left|\Delta^{2}\right|$ as a homotopy from the edge $(0,2)$ to the edge path $(0,1)(1,2)$, we obtain a homotopy from the composite map $j t$,

$$
\left|i . \mathbf{E}_{0}\right| \stackrel{t}{\longrightarrow}\left|i . \mathbf{B}_{0}\right| \stackrel{j}{\longrightarrow} \Omega|i . S . \mathbf{B}|_{(2)},
$$

to the loop product of two composite maps $j s$ and $j q$. But in the $H$-space $\Omega|i . S . \mathbf{B}|$ the loop product is homotopic to the composition law, by a well-known fact about loop spaces of $H$-spaces, whence the observation as stated.

The same consideration leads, more generally, to the following. 
Observation. For every $n \geq 0$, the following two composite maps are homotopic:

$$
\left|i . S .{ }^{n} \mathbf{E}\right| \underset{s \vee q}{\stackrel{t}{\longrightarrow}}\left|i . S .{ }^{n} \mathbf{B}\right| \longrightarrow \Omega\left|i . S .{ }^{n+1} \mathbf{B}\right| .
$$

Theorem 6.5. The additivity theorem is valid (i.e., each of the equivalent conditions of Proposition 6.4 is valid) if in the definition of the K-theory, $\Omega|i . S . \mathbf{B}|$ is replaced with $\Omega^{\infty}\left|i . S .{ }^{\infty} \mathbf{B}\right|=\lim _{n} \Omega^{n}\left|i . S .{ }^{n} \mathbf{B}\right|$.

Proof. First, we observe that Proposition 6.4 is formal in the sense that it also applies to the new definition of the $K$-theory. By the preceding observation, the composite maps

$$
\Omega^{\infty}\left|i . S .{ }^{\infty} \mathbf{E}\right| \underset{s \vee q}{\stackrel{t}{\longrightarrow}} \Omega^{\infty}\left|i . S .{ }^{\infty} \mathbf{B}\right| \longrightarrow \Omega^{\infty}\left|i . S .{ }^{\infty} \mathbf{B}\right|
$$

are homotopic. Since the map on the right is an isomorphism, this is one of the equivalent conditions of the additivity theorem (Proposition 6.4).

Remark. As a consequence of the theorem, we could add yet another reformulation of the additivity theorem to the list of Proposition 6.4 (see also Theorem 6.6). Namely, the additivity theorem as stated there implies that the maps $\left|i . S .{ }^{n} \mathbf{B}\right| \longrightarrow \Omega\left|i . S .{ }^{n+1} \mathbf{B}\right|$ are homotopy equivalences for $n \geq 1$. Conversely, if these maps are homotopy equivalences, then so is $\Omega|i . S . \mathbf{B}| \longrightarrow \Omega^{\infty}\left|i . S .{ }^{\infty} \mathbf{B}\right|$, and thus the additivity theorem is provided by Theorem 6.5.

Let $F: \mathbf{A} \longrightarrow \mathbf{B}$ be a right exact functor between two left systems of diagram categories or between two left pointed dérivateurs. We denote by $\mathbf{S} .(F: \mathbf{A} \longrightarrow \mathbf{B})$ the fiber product of the diagram

$$
\mathbf{S . A} \stackrel{F}{\longrightarrow} \mathbf{S . B} \stackrel{\partial_{0}}{\longleftarrow} P \mathbf{S . B},
$$

where $\partial_{0}=d_{0}^{*}$ is the map induced by $d_{0}: \Delta^{n} \longrightarrow \Delta^{n+1}$. By Proposition 1.3, S. $(F$ : $\mathbf{A} \longrightarrow \mathbf{B})$ is a simplicial left system of diagram categories or a simplicial left pointed dérivateur. Thus, for every $n$ we have a commutative diagram

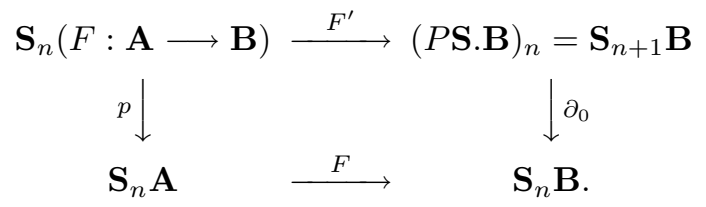

By construction, we can identify an object of $\mathbf{S}_{n}(F: \mathbf{A} \longrightarrow \mathbf{B})$ ? with a triple $(A, c, B)$ the objects in which are in $\mathbf{S}_{n} \mathbf{A}_{\text {? }}$ and $\mathbf{S}_{n+1} \mathbf{B}_{\text {? }}$, respectively, together with an isomorphism $F A \stackrel{c}{\simeq} \partial_{0} B$. We note that all the maps in the above diagram are right exact.

Let $G: \mathbf{B} \longrightarrow \mathbf{S}_{n+1} \mathbf{B}$ be the morphism constructed above. We have $\partial_{0} G B=0$, and $G$ factors as $F^{\prime} \circ G^{\prime}$, where $G^{\prime}: \mathbf{B} \longrightarrow \mathbf{S}_{n}(F: \mathbf{A} \longrightarrow \mathbf{B}), B \stackrel{G^{\prime}}{\longmapsto}(0,1, G B)$.

Regarding $\mathbf{B}$ as a simplicial object in a trivial way, we obtain a sequence

$$
\mathbf{B} \stackrel{G^{\prime}}{\longrightarrow} \mathbf{S} .(F: \mathbf{A} \longrightarrow \mathbf{B}) \stackrel{p}{\longrightarrow} \mathbf{S} . \mathbf{A},
$$

in which the composition map is trivial. This leads to a sequence

$$
i . S . \mathbf{B} \longrightarrow i . S . S .(\mathbf{A} \longrightarrow \mathbf{B}) \longrightarrow i . S . S . \mathbf{A},
$$

induced by (9).

Similarly, there is a sequence

$$
i . S . \mathbf{B} \longrightarrow P(i . S . S . \mathbf{B}) \longrightarrow i . S . S . \mathbf{B},
$$

where " $P$ " refers, say, to the first $S$-direction. 
Theorem 6.6. The following statements are equivalent:

1) the additivity theorem (Proposition 6.4) is valid;

2) the sequence

$$
i . S . \mathbf{B} \longrightarrow i . S . S .(\mathbf{A} \longrightarrow \mathbf{B}) \longrightarrow i . S . S . \mathbf{A}
$$

is a fibration up to homotopy;

3) the sequence

$$
i . S . \mathbf{B} \longrightarrow P(i . S . S . \mathbf{B}) \longrightarrow i . S . S . \mathbf{B}
$$

is a fibration up to homotopy;

4) the map $\left|i . S .{ }^{n} \mathbf{B}\right| \longrightarrow \Omega\left|i . S \cdot{ }^{n+1} \mathbf{B}\right|$ is a homotopy equivalence for any $n \geq 1$.

If the equivalent conditions 1)-4) are fulfilled, then the spectrum $n \mapsto i . S .{ }^{n} \mathbf{B}$, with structural maps defined as the map $\left|i . \mathbf{B}_{0}\right| \longrightarrow \Omega|i . S . \mathbf{B}|$ above, is an $\Omega$-spectrum beyond the first term. The spectrum is connected (the $n$th term is $(n-1)$-connected). As a consequence, the $K$-theory for $\mathbf{B}$ can be defined equivalently as the space

$$
\Omega^{\infty}\left|i . S .{ }^{\infty} \mathbf{B}\right|=\lim _{n} \Omega^{n}\left|i . S .{ }^{n} \mathbf{B}\right| .
$$

Proof. 3) is a consequence of 2$)$. Since the space $|P(i . S . S . \mathbf{B})|$ is contractible, condition 3) implies that the map $|i . S . \mathbf{B}| \longrightarrow \Omega|i . S . S . \mathbf{B}|$ is a homotopy equivalence, and more generally, the map $\left|i . S .{ }^{n} \mathbf{B}\right| \longrightarrow \Omega\left|i . S .{ }^{n+1} \mathbf{B}\right|, n \geq 1$, is also a homotopy equivalence. Therefore, 4) follows from 3). By the second observation after Proposition 6.4, the two composition maps

$$
|i . S . \mathbf{E}| \underset{s \vee q}{\stackrel{t}{\longrightarrow}}|i . S . \mathbf{B}| \longrightarrow \Omega|i . S . S . \mathbf{B}|
$$

are homotopic. If the map on the right is a homotopy equivalence, then $t$ is homotopic to $s \vee q$. Thus, 4) implies 1). It remains to check that 1) $\Longrightarrow 2$ ).

By Lemma 4.2, it suffices to show that for every $n$ the sequence $i . S . \mathbf{B} \longrightarrow i . S . S_{n}(\mathbf{A} \longrightarrow$ B) $\longrightarrow i . S . S_{n} \mathbf{A}$ is a fibration (because the base term i.S.S $\mathbf{A}$ is connected for every $n)$. Using the additivity theorem, we shall show that this sequence is the same, up to homotopy, as the trivial fibration sequence associated with the product $i . S . \mathbf{B} \times i . S . S_{n} \mathbf{A}$.

Consider the maps $u: \Delta^{1} \longrightarrow \Delta^{n+1}, 0 ; 1 \mapsto 0 ; 1$, and $v: \Delta^{n+1} \longrightarrow \Delta^{1}, 0 \mapsto 0, i \mapsto 1$ if $i \geq 1$. Then $u$ is left adjoint to $v$. To simplify the notation, the corresponding maps $\operatorname{Ar} \Delta^{1} \longrightarrow \operatorname{Ar} \Delta^{n+1}$ and $\operatorname{Ar} \Delta^{n+1} \longrightarrow \operatorname{Ar} \Delta^{1}$ induced by $u$ and $v$ are denoted by the same letters. Let $\overline{\mathbf{B}}=\left\{v^{*} u^{*} B \mid B \in \mathbf{S}_{n+1} \mathbf{B}\right\}$; then $v^{*} u^{*} B_{(0,0)}=B_{(0,0)}, v^{*} u^{*} B_{(0, i)}=B_{(0,1)}$ for any $1 \leq i \leq n+1$ and $v^{*} u^{*} B_{(i, j)}=B_{(1,1)}$ for any $i \geq 1$.

Let $\overline{\overline{\mathbf{B}}}=\left\{\sigma_{0} \partial_{0} B \mid B \in \mathbf{S}_{n+1} \mathbf{B}\right\}$, where $\sigma_{0}: \mathbf{S}_{n} \mathbf{B} \longrightarrow \mathbf{S}_{n+1} \mathbf{B}$ is the functor induced by $s_{0}: \operatorname{Ar} \Delta^{n+1} \longrightarrow \operatorname{Ar} \Delta^{n}$. Note that $\sigma_{0}$ is right adjoint to $\partial_{0}$.

Let $m: \Delta^{1} \times \operatorname{Ar} \Delta^{n+1} \longrightarrow \operatorname{Ar} \Delta^{n+1}$ be the map taking $(0,(i, j))$ to $(u v(i), u v(j))$ and $(1,(i, j))$ to $(i, j)$. Then $m^{*}$ takes an object $B \in \mathbf{S}_{n+1} \mathbf{B}_{\text {? }}$ to the object in $\mathbf{S}_{n+1} \mathbf{B}_{\Delta^{1} \times}$ ? that is depicted in $\mathbf{S}_{n+1} \mathbf{B}_{\text {? }}$ as the adjunction morphism $v^{*} u^{*} B \longrightarrow B$.

Next, let $l: \Delta^{1} \times \operatorname{Ar} \Delta^{n+1} \longrightarrow \operatorname{Ar} \Delta^{n+1}$ be the map taking $(0,(i, j))$ to $(i, j)$ and $(1,(i, j))$ to $\left(d_{0} s_{0}(i), d_{0} s_{0}(j)\right)$. Then $l^{*}$ takes an object $B \in \mathbf{S}_{n+1} \mathbf{B}_{\text {? }}$ to the object in $\mathbf{S}_{n+1} \mathbf{B}_{\Delta^{1} \times \text { ? }}$ that is evaluated as $B$ at 0 , and as $\sigma_{0} \partial_{0} B$ at 1 . This object is depicted in $\mathbf{S}_{n+1} \mathbf{B}_{\text {? }}$ as the adjunction morphism $\beta: B \longrightarrow \sigma_{0} \partial_{0} B$.

The restriction of the right exact morphism $\left(1_{\Delta^{1}} \times m\right)^{*} l^{*}: \mathbf{B}\left(\operatorname{Ar} \Delta^{n+1}\right) \longrightarrow \mathbf{B}(\square \times$ $\left.\Delta^{n+1}\right)$ to $\mathbf{S}_{n+1} \mathbf{B}$ takes an object $B \in \mathbf{S}_{n+1} \mathbf{B}_{\text {? }}$ to an object in $\mathbf{E}\left(\operatorname{Ar} \Delta^{n+1}\right)$ ? $\subset \mathbf{B}(\square \times$ $\left.\operatorname{Ar} \Delta^{n+1}\right)$ ?. Thus, we obtain a right exact functor

$$
T: \mathbf{S}_{n+1} \mathbf{B} \stackrel{l^{*}}{\longrightarrow} \mathbf{S}_{n+1} \mathbf{B}\left(\Delta^{1}\right) \stackrel{\left(1_{\Delta^{1}} \times m\right)^{*}}{\longrightarrow} \mathbf{E}\left(\operatorname{Ar} \Delta^{n+1}\right)
$$

such that $t \circ T$ is the identity morphism on $\mathbf{S}_{n+1} \mathbf{B}$ and $s \circ T(B) \in \overline{\mathbf{B}}, q \circ T(B) \in \overline{\overline{\mathbf{B}}}$ for every $B \in \mathbf{S}_{n+1} \mathbf{B}$. So, $T$ takes its values in $\mathbf{E}\left(\overline{\mathbf{B}}, \mathbf{S}_{n+1} \mathbf{B}, \overline{\overline{\mathbf{B}}}\right)$, and actually this is a 
functor

$$
T: \mathbf{S}_{n+1} \mathbf{B} \longrightarrow \mathbf{E}\left(\overline{\mathbf{B}}, \mathbf{S}_{n+1} \mathbf{B}, \overline{\overline{\mathbf{B}}}\right) .
$$

To illustrate the above procedure, let us temporarily think of $\mathbf{S}_{n+1} \mathbf{B}$ as "strings" $\mathbf{B}\left(\Delta^{n}\right)$ via the equivalence $\ell^{*}: \mathbf{S}_{n+1} \mathbf{B} \longrightarrow \mathbf{B}\left(\Delta^{n}\right)$ (see Proposition 3.1). Consider the function $\widetilde{m}: \Delta^{1} \times \Delta^{n} \longrightarrow \Delta^{n}$ defined by

$$
(0, i) \longmapsto 0, \quad(1, i) \longmapsto i .
$$

Then the induced right exact morphism $\widetilde{m}^{*}: \mathbf{B}\left(\Delta^{n}\right) \longrightarrow \mathbf{B}\left(\Delta^{1} \times \Delta^{n}\right)$ takes an object $B \in \mathbf{B}\left(\Delta^{n}\right)$ ? to the object in $\mathbf{B}\left(\Delta^{1} \times \Delta^{n}\right)$ ? that is depicted in $\mathbf{B}_{\text {? }}$ as

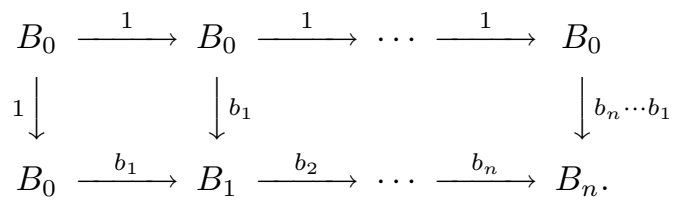

Let $k: \Delta^{1} \times \Delta^{n} \longrightarrow$ Ar $\Delta^{n+1}$ be the map $(i, j) \longmapsto(i, j+1)$, and let $\alpha_{j}: \square \longrightarrow \Delta^{1} \times \Delta^{n}$ be the map taking $(0 ; 1,0)$ to $(0 ; 1, j)$ and $(0 ; 1,1)$ to $(0 ; 1, j+1)$. Denote by $\mathbf{S}_{n+1}^{\prime} \mathbf{B}$ the left subsystem of diagram categories or the left pointed subdérivateur of $\mathbf{B}\left(\Delta^{1} \times \Delta^{n}\right)$ consisting of the objects $B$ such that all the squares $\alpha_{j}^{*} B, j \leq n$, are co-Cartesian and $B_{(1,0)}=O$ is a zero object. Then the restriction morphisms $k^{*}: \mathbf{S}_{n+1} \mathbf{B} \longrightarrow \mathbf{S}_{n+1}^{\prime} \mathbf{B}$ and $w^{*}: \mathbf{S}_{n+1}^{\prime} \mathbf{B} \longrightarrow \mathbf{B}\left(\Delta^{n}\right)$ with $w: \Delta^{n} \longrightarrow \Delta^{1} \times \Delta^{n}, j \longmapsto(0, j)$ are easily seen to be equivalences.

The restriction of the morphism $\left(1_{\Delta^{1}} \times \widetilde{m}\right)^{*}: \mathbf{B}\left(\Delta^{1} \times \Delta^{n}\right) \longrightarrow \mathbf{B}\left(\square \times \Delta^{n}\right)$ to $\mathbf{S}_{n}^{\prime} \mathbf{B}$ takes an object $B \in \mathbf{S}_{n}^{\prime} \mathbf{B}_{\text {? }}$ to the object in $\mathbf{B}\left(\square \times \Delta^{n}\right)$ ? that is depicted in $\mathbf{B}_{\text {? }}$ as

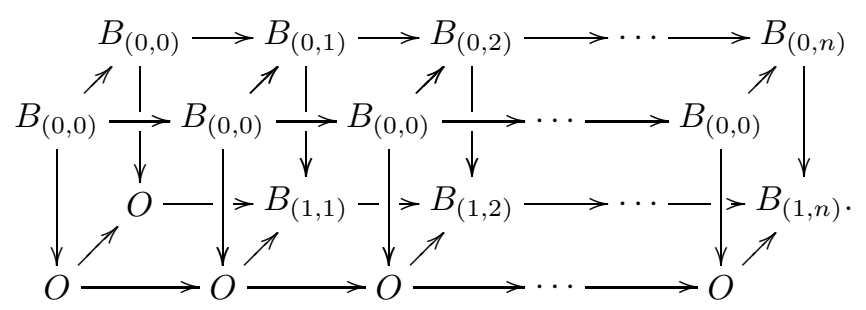

The back wall of the diagram is the element $B$ of $\mathbf{S}_{n}^{\prime} \mathbf{B}_{\text {? }}$ drawn in $\mathbf{B}_{\text {? }}$. We obtain a right exact morphism $\widetilde{T}=\left(1_{\Delta^{1}} \times \widetilde{m}\right)^{*} \circ w^{*-1}: \mathbf{B}\left(\Delta^{n}\right) \longrightarrow \mathbf{E}\left(\Delta^{n}\right)$, because each transverse $x$ th square $B_{x, \square}, x \leq n$, is co-Cartesian.

Now, we return to the morphism $T$ and lift it to $\mathbf{S}_{n}(\mathbf{A} \longrightarrow \mathbf{B})$. More precisely, we send an object $(A, c, B) \in \mathbf{S}_{n}(\mathbf{A} \longrightarrow \mathbf{B})_{\text {? }}$ to $\left(\partial_{0} T \sigma_{0} A, \partial_{0} T \sigma_{0}(c), T B\right) \in \mathbf{E}\left(\mathbf{S}_{n}(\mathbf{A} \longrightarrow \mathbf{B})\right)_{\text {? }}$. We use the commutative diagram

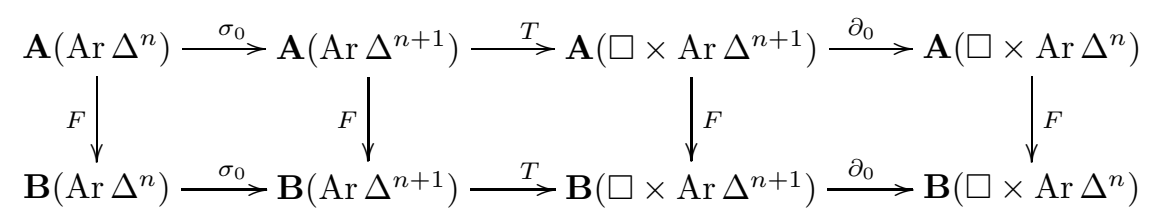

to show that $F \partial_{0} T \sigma_{0} A=\partial_{0} T \sigma_{0} F A$. The relation $\partial_{0} T B=\partial_{0} T \sigma_{0} \partial_{0} B$ is straightforward.

Put $\mathbf{B}^{\prime}=\left\{\left(\partial_{0} v^{*} u^{*} \sigma_{0} A, \partial_{0} v^{*} u^{*} \sigma_{0}(c), v^{*} u^{*} B\right) \mid(A, c, B) \in \mathbf{S}_{n}(\mathbf{A} \longrightarrow \mathbf{B})\right\}$ and $\mathbf{B}^{\prime \prime}=$ $\left\{\left(A, c, \sigma_{0} \partial_{0} B\right) \mid(A, c, B) \in \mathbf{S}_{n}(\mathbf{A} \longrightarrow \mathbf{B})\right\}$.

We obtain a right exact functor

$$
T^{\prime}: \mathbf{S}_{n}(\mathbf{A} \longrightarrow \mathbf{B}) \longrightarrow \mathbf{E}\left(\mathbf{B}^{\prime}, \mathbf{S}_{n}(\mathbf{A} \longrightarrow \mathbf{B}), \mathbf{B}^{\prime \prime}\right),
$$

where $s \circ T^{\prime}$ sends $(A, c, B)$ to $\left(\partial_{0} v^{*} u^{*} \sigma_{0} A, \partial_{0} v^{*} u^{*} \sigma_{0}(c), v^{*} u^{*} B\right), t \circ T^{\prime}$ being the identity, 
and $q \circ T^{\prime}$ sends $(A, c, B)$ to $\left(A, c, \sigma_{0} \partial_{0} B\right)$. Thus we get a right exact sequence $s \circ T^{\prime} \longrightarrow$ $1 \longrightarrow q \circ T^{\prime}$. By our assumption, the map

$$
\left(s \circ T^{\prime}, q \circ T^{\prime}\right): S . S_{n}(\mathbf{A} \longrightarrow \mathbf{B}) \longrightarrow S . \mathbf{B}^{\prime} \times S . \mathbf{B}^{\prime \prime}
$$

is a homotopy equivalence, with a homotopy inverse induced by the coproduct.

Clearly, the morphism $\mathbf{B}^{\prime} \longrightarrow \mathbf{B}$ taking $\left(\partial_{0} v^{*} u^{*} \sigma_{0} A, \partial_{0} v^{*} u^{*} \sigma_{0}(c), v^{*} u^{*} B\right)$ to $B_{(0,1)}$ is an equivalence. A quasiinverse to it is $G^{\prime}$.

We show that the morphism $\delta: \mathbf{S}_{n} \mathbf{A} \longrightarrow \mathbf{B}^{\prime \prime}, A \mapsto\left(A, 1, \sigma_{0} F A\right)$, is a quasinverse to the restriction of $p$ to $\mathbf{B}^{\prime \prime}$. Obviously, $\delta$ is faithful. For any object $(A, c, B) \in \mathbf{B}^{\prime \prime}$, the morphism $\left(1, \sigma_{0}(c)\right):\left(A, 1, \sigma_{0} F A\right) \longrightarrow(A, c, B)$ is an isomorphism. Also, every morphism $(a, b): \delta A \longrightarrow \delta A^{\prime}$ in $\mathbf{B}^{\prime \prime}$ is equal to $\left(a, \sigma_{0} F a\right)$, whence $\delta$ is full. We see that $\delta$ is an equivalence.

So, the map $i . S . \mathbf{B}^{\prime} \times i . S . \mathbf{B}^{\prime \prime} \longrightarrow i . S . \mathbf{B} \times i . S . S_{n} \mathbf{A}$ is a homotopy equivalence. Hence, so is the composition

$$
i . S . S_{n}(\mathbf{A} \longrightarrow \mathbf{B}) \longrightarrow i . S . \mathbf{B}^{\prime} \times i . S . \mathbf{B}^{\prime \prime} \longrightarrow i . S . \mathbf{B} \times i . S . S_{n} \mathbf{A} .
$$

This latter homotopy equivalence fits into the following commutative diagram:

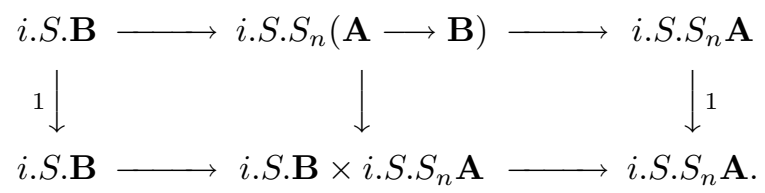

Being homotopy equivalent to the trivial fibration (the lower row of the diagram), the upper sequence is a fibration, as required.

Remark. Let $\Im$ be a class of left systems of diagram categories or left pointed dérivateurs satisfying the following two conditions:

1) $\mathbf{B} \in \Im$ implies $\mathbf{S}_{n} \mathbf{B} \in \Im$ for any $n$;

2) the map $i . S . \mathbf{E} \stackrel{(s, q)}{\longrightarrow} i . S . \mathbf{B} \times i . S . \mathbf{B}$ is a homotopy equivalence for any $\mathbf{B} \in \Im$.

The proof of Theorem 6.6 then shows that the spectrum

$$
n \longmapsto i . S .{ }^{n} \mathbf{B}
$$

is an $\Omega$-spectrum beyond the first term, so that the $K$-theory for every $\mathbf{B} \in \Im$ can be defined equivalently as the space

$$
\Omega^{\infty}\left|i . S .{ }^{\infty} \mathbf{B}\right|=\lim _{n} \Omega^{n}\left|i . S .{ }^{n} \mathbf{B}\right| .
$$

A left pointed dérivateur $\mathbf{D}$ with domain Ord is said to be complicial if for some complicial bi-Waldhausen category $\mathcal{C}$ in the sense of Thomason [19] there is a right exact equivalence $F: \mathbf{D C} \longrightarrow \mathbf{D}$ that is closed under formation of canonical homotopy pushouts and canonical homotopy pullbacks. In this case we say that $\mathbf{D}$ is represented by $\mathcal{C}$. That equivalence induces a homotopy equivalence of bisimplicial sets $F: i . S . \mathbf{C} \longrightarrow i . S . \mathbf{D}$.

Theorem 6.7 ([10]). The class of complicial dérivateurs satisfies the conditions of the remark above.

Proposition 6.8. Under the assumptions of Theorem 6.6, suppose we are given a sequence $\mathbf{A} \longrightarrow \mathbf{B} \longrightarrow \mathbf{C}$ of right exact morphisms between left systems of diagram categories or left pointed dérivateurs. Then the square

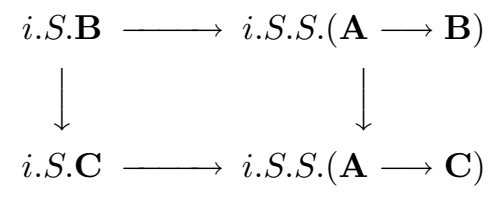

is homotopy Cartesian. 
Proof. There is a commutative diagram

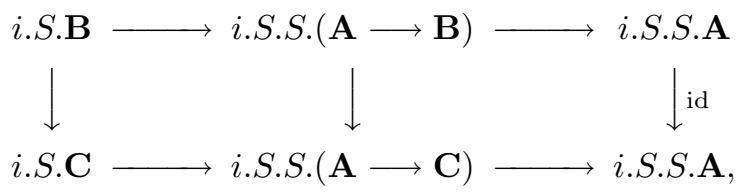

in which the rows are fibrations up to homotopy by Theorem 6.6. Therefore, the square on the left is homotopy Cartesian.

Corollary 6.9. Under the assumptions of Theorem 6.6, the following two assertions are valid.

1) Every right exact morphism gives rise to a fibration

$$
i . S . \mathbf{B} \longrightarrow i . S . \mathbf{C} \longrightarrow i . S . S .(\mathbf{B} \longrightarrow \mathbf{C}) \text {. }
$$

2) If $\mathbf{C}$ is a retract of $\mathbf{B}$ (by right exact functors), then there is a splitting

$$
i . S . \mathbf{B} \simeq i . S . \mathbf{C} \times i . S . S .(\mathbf{C} \longrightarrow \mathbf{B}) .
$$

Proof. 1). If $\mathbf{A}=\mathbf{B}$, then the space $|i . S . S .(\mathbf{A}=\mathbf{A})|$ is contractible, whence the first assertion.

2). This is a consequence of Proposition 6.8, because the composition $\mathbf{A} \longrightarrow \mathbf{B} \longrightarrow \mathbf{C}$ is an identity map, and then $i . S . S .(\mathbf{A} \longrightarrow \mathbf{C})$ is contractible.

\section{$\S 7$. Comparison theOrem}

Given an exact category $\mathcal{E}$, we would like to compare Quillen's $K$-theory $K(\mathcal{E})$ of $\mathcal{E}$ with the $K$-theory of the associated bidérivateur $\mathbf{D}^{b}(\mathcal{E})$.

Let $w C^{b}(\mathcal{E})$ denote the Waldhausen category of quasiisomorphisms in $C^{b}(\mathcal{E})$ whose cofibrations are componentwise admissible monomorphisms. For every $I \in \operatorname{Dirf}$ we have a natural functor

$$
\text { Ho : } C^{b}\left(\mathcal{E}^{I}\right) \longrightarrow D^{b}\left(\mathcal{E}^{I}\right) \text {. }
$$

The image under the functor Ho of any co-Cartesian square of $C^{b}(\mathcal{E})^{\square}=C^{b}\left(\mathcal{E}^{\square}\right)$

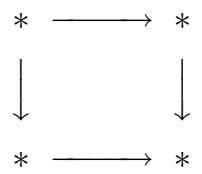

in which the horizontal arrows are cofibrations is a co-Cartesian square in $\mathbf{D}^{b}(\mathcal{E})_{\square}$ (this is dual to $[16,3.14])$. Therefore, Ho induces a map of bisimplicial objects $\nu: w \cdot S . C^{b}(\mathcal{E}) \longrightarrow$ $i . S . \mathbf{D}^{b}(\mathcal{E})$. Consider the map

$$
K(\tau): K(\mathcal{E}) \longrightarrow K\left(w C^{b}(\mathcal{E})\right)
$$

induced by the map $\tau$ taking an object of $\mathcal{E}$ to the complex concentrated at the zeroth degree $\left(K\left(w C^{b}(\mathcal{E})\right)\right.$ stands for the Waldhausen $K$-theory of $\left.w C^{b}(\mathcal{E})\right)$.

The first Maltsiniotis conjecture [7]. The map $K(\rho)=K(\nu \tau): K(\mathcal{E}) \longrightarrow K\left(\mathbf{D}^{b}(\mathcal{E})\right)$ is a homotopy equivalence.

The homomorphism $K_{0}(\mathcal{E}) \longrightarrow K_{0}\left(\mathbf{D}^{b}(\mathcal{E})\right)$ is an isomorphism, since the Grothendieck groups $K_{0}(\mathcal{E})$ and $K_{0}\left(D^{b}(\mathcal{E})\right)$ are naturally isomorphic (an exercise!) and $K_{0}\left(\mathbf{D}^{b}(\mathcal{E})\right.$ ) is naturally isomorphic to $K_{0}\left(D^{b}(\mathcal{E})\right)$ by Lemma 3.5.

The first Maltsiniotis conjecture is very resistant in general. However, some information can be obtained for a large class of exact categories including the Abelian categories. The following statement shows that Quillen's $K$-theory $K(\mathcal{E})$ of an exact category $\mathcal{E}$ in this class is a retract of $K\left(\mathbf{D}^{b}(\mathcal{E})\right)$. 
Theorem 7.1. Let $\mathcal{E}$ be an extension closed and full exact subcategory of an Abelian category $\mathcal{A}$ satisfying the conditions of the resolution theorem. That is,

(1) if $0 \longrightarrow M^{\prime} \longrightarrow M \longrightarrow M^{\prime \prime} \longrightarrow 0$ is exact in $\mathcal{A}$ and $M, M^{\prime \prime} \in \mathcal{E}$, then $M^{\prime} \in \mathcal{E}$ and

(2) for any object $M \in \mathcal{A}$ there is a finite resolution $0 \longrightarrow P_{n} \longrightarrow P_{n-1} \longrightarrow \cdots \longrightarrow$ $P_{0} \longrightarrow M \longrightarrow 0$ with $P_{i} \in \mathcal{E}$.

Then a natural map

$$
K(\rho): K(\mathcal{E}) \longrightarrow K\left(\mathbf{D}^{b}(\mathcal{E})\right)
$$

is such that for some $p: K\left(\mathbf{D}^{b}(\mathcal{E})\right) \longrightarrow K(\mathcal{E})$ the composition $p \circ K(\rho)$ is homotopic to the identity map. In particular, each $K$-group $K_{n}(\mathcal{E})$ is a direct summand of $K_{n}\left(\mathbf{D}^{b}(\mathcal{E})\right)$.

We postpone the proof. It should be noted that it depends substantially on Neeman's results [22] on $K$-theory for triangulated categories.

In $[24,25]$ it was shown that the natural morphism $K(\mathcal{C}) \longrightarrow K(\mathbf{D C})$ from the Waldhausen $K$-theory to the $K$-theory of its dérivateur cannot be an equivalence in general. For instance, this is not an equivalence for the Waldhausen $K$-theory of spaces. This does not mean, however, that the $K$-groups $K_{n}(\mathcal{C})$ cannot be recovered from its dérivateur, and that this is a counterexample to the comparison problem stated above for exact categories.

A good Waldhausen category is a Waldhausen category that can be embedded in the category of cofibrant objects of a pointed model category, and whose Waldhausen structure is induced by the ambient model structure (see the precise definition in [25]). Though there exist nongood Waldhausen categories (see [25, Example 2.2]), in practice it turns out that, given a Waldhausen category, we always have a good Waldhausen model, i.e., a good Waldhausen category with the same $K$-theory space up to homotopy. Any good Waldhausen category is a Waldhausen category of cofibrant objects; therefore it gives rise to a left pointed dérivateur $\mathbf{D C}$ (Theorem 2.8). The following theorem is also a consequence of a result by Cisinski and Toën [24, Subsection 2.16].

Theorem 7.2 (first stated by Toën [26]). Let $\mathcal{C}$ and $\mathcal{C}^{\prime}$ be two good Waldhausen categories such that their associated dérivateurs $\mathbf{D C}$ and $\mathbf{D} \mathcal{C}^{\prime}$ are equivalent. Then the Waldhausen $K$-theory spectra $K(\mathcal{C})$ and $K\left(\mathcal{C}^{\prime}\right)$ are also equivalent.

With certain extra data, $\mathbf{B}$ encodes the structure of a triangulated category on $\mathbf{B}_{0}$; see $[16,5,6,7]$. This structure is carried over canonically to all the categories $\mathbf{B}_{I}$, $I \in \mathcal{D}$ ia. In this case $\mathbf{B}$ is referred to as a system of triangulated diagram categories or triangulated dérivateur, respectively. The following result shows that such a $\mathbf{B}$ contains strictly more information than its triangulated category $\mathbf{B}_{0}$.

Proposition 7.3. There exist two nonequivalent triangulated dérivateurs $\mathbf{B}$ and $\mathbf{B}^{\prime}$ whose associated triangulated categories $\mathbf{B}_{0}$ and $\mathbf{B}_{0}^{\prime}$ are equivalent.

Proof. Let $\mathcal{C}=m \mathcal{M}\left(\mathbf{Z} / p^{2}\right)$ and $\mathcal{C}^{\prime}=m \mathcal{M}\left(\mathbf{Z} / p[\varepsilon] / \varepsilon^{2}\right)$ be two stable model categories considered in [27]. Here $\mathcal{M}\left(\mathbf{Z} / p^{2}\right)$ and $\mathcal{M}\left(\mathbf{Z} / p[\varepsilon] / \varepsilon^{2}\right)$ denote the corresponding categories of finitely generated modules. Since $\mathbf{Z} / p^{2}$ and $\mathbf{Z} / p[\varepsilon] / \varepsilon^{2}$ are quasi-Frobenius rings, it follows that $\mathcal{M}\left(\mathbf{Z} / p^{2}\right)$ and $\mathcal{M}\left(\mathbf{Z} / p[\varepsilon] / \varepsilon^{2}\right)$ are Frobenius categories, and $\mathbf{D C}$ and $\mathbf{D} \mathcal{C}^{\prime}$ are triangulated dérivateurs by [16, Subsection 4.19]. From [27, Subsection 1.4] we know that $\mathbf{D} \mathcal{C}_{0}$ and $\mathbf{D} \mathcal{C}_{0}^{\prime}$ are equivalent as triangulated categories. But the dérivateurs $\mathbf{D} \mathcal{C}$ and $\mathbf{D} \mathcal{C}^{\prime}$ cannot be equivalent by Theorem 7.2 , because the Waldhausen $K$-theories $K(\mathcal{C})$ and $K\left(\mathcal{C}^{\prime}\right)$ are nonequivalent by [27, Subsection 1.7].

Another problem arising in our context (see also [7, Conjecture 2]) is the localization theorem. Suppose we are given a family $\mathcal{W}=\left\{\mathcal{W}_{I} \subseteq\right.$ Mor $\mathbf{B}_{I} \mid I \in \mathcal{D}$ ia $\}$ of morphisms compatible with the structure functors $f^{*}$ and $f_{!}$, i.e., $f^{*}\left(\mathcal{W}_{J}\right) \subseteq \mathcal{W}_{I}$ and $f_{!}\left(\mathcal{W}_{I}\right) \subseteq \mathcal{W}_{J}$ 
for every map $f: I \longrightarrow J$. Let $\mathbf{B}_{\text {? }}\left[\mathcal{W}_{?}^{-1}\right]$ denote the category of fractions obtained by inverting the maps in $\mathcal{W}_{\text {? }}$. We also require the following condition to be fulfilled: a morphism is in $\mathcal{W}_{\text {? }}$ if and only if its image in $\mathbf{B}_{\text {? }}\left[\mathcal{W}_{?}^{-1}\right]$ is an isomorphism. Suppose the hyperfunctor

$$
I \stackrel{Q}{\longmapsto} \mathbf{B}_{I}\left[\mathcal{W}_{I}^{-1}\right]
$$

determines a left system of diagram categories or a left pointed dérivateur, respectively. We denote it by $\mathbf{B}\left[\mathcal{W}^{-1}\right]$. Suppose further that the quotient morphism $Q: \mathbf{B} \longrightarrow \mathbf{B}\left[\mathcal{W}^{-1}\right]$ is right exact.

In the case where $\mathbf{B}$ is a system of triangulated diagram categories or, respectively, a triangulated dérivateur, any thick subcategory $\mathbf{A}_{0}$ of $\mathbf{B}_{0}$ gives rise to a localization in $\mathbf{B}$. Namely, given $I \in \mathcal{D}$ ia, we can put $\mathbf{A}_{I}=\left\{A \in \mathbf{B}_{I} \mid A_{x} \in \mathbf{A}_{0}\right.$ for all $\left.x \in I\right\}$. Then $\mathbf{A}_{I}$ is thick in $\mathbf{B}_{I}$, and the functor $I \longmapsto \mathbf{A}_{I}$ determines a system of triangulated diagram categories or a triangulated dérivateur, and the quotient is then constructed in a natural way (see [5, p. 39]).

The second Maltsiniotis conjecture [7]. Suppose we are given a sequence of morphisms between left systems of diagram categories or left pointed dérivateurs,

$$
\mathbf{A} \stackrel{F}{\longrightarrow} \mathbf{B} \stackrel{Q}{\longrightarrow} \mathbf{B}\left[\mathcal{W}^{-1}\right]
$$

where $Q$ is the quotient morphism and $F$ is a right exact equivalence between $\mathbf{A}$ and $Q^{-1}(0)=\left\{B \in \mathbf{B}_{?} \mid 0 \longrightarrow B \in \mathcal{W}_{?}\right\}$. Then the induced sequence of $K$-theory spaces

$$
K(\mathbf{A}) \longrightarrow K(\mathbf{B}) \longrightarrow K\left(\mathbf{B}\left[\mathcal{W}^{-1}\right]\right)
$$

is a fibration up to homotopy.

With the morphism $F$ we have already associated a fibration (see Corollary 5.4(1)):

$$
i . S . \mathbf{A} \longrightarrow i . S . \mathbf{B} \longrightarrow i . N . S .(\mathbf{A} \longrightarrow \mathbf{B}) \text {. }
$$

There is a natural map from i.N.S. $(\mathbf{A} \longrightarrow \mathbf{B})$ to $i . S . \mathbf{B}\left[\mathcal{W}^{-1}\right]$. Therefore, the localization theorem reduces, say, to showing that the latter map is a homotopy equivalence.

It remains to prove Theorem 7.1, as promised. We start with preparations.

Definition. An additive category $\mathcal{T}$ will be called a category with squares provided

$\diamond \mathcal{T}$ has an automorphism $\Sigma: \mathcal{T} \longrightarrow \mathcal{T}$;

$\diamond \mathcal{T}$ is equipped with a collection of special squares

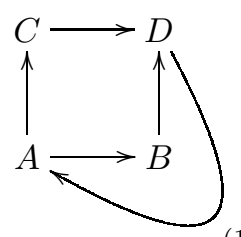

This means that the square

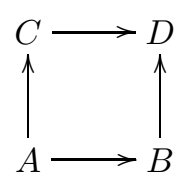

is commutative in $\mathcal{T}$, and there is a map $D \longrightarrow \Sigma A$ depicted as the curly arrow. The mark (1) in the label of the arrow is to remind us that this map is of degree 1, i.e., a map $D \longrightarrow \Sigma A$.

For two categories with squares, a special functor

$$
F: \mathcal{S} \longrightarrow \mathcal{T}
$$


is an additive functor such that there is a natural isomorphism $\Sigma F \simeq F \Sigma$ and $F$ takes special squares in $\mathcal{S}$ to special squares in $\mathcal{T}$.

If $\mathcal{T}$ is a category with squares, by the fold of the square

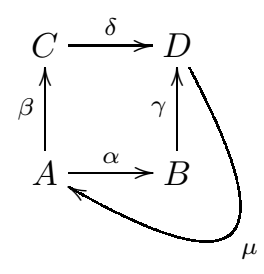

we shall mean the sequence

$$
A \stackrel{(\alpha,-\beta)^{t}}{\longrightarrow} B \oplus C \stackrel{(\gamma, \delta)}{\longrightarrow} D \stackrel{\mu}{\longrightarrow} \Sigma A .
$$

Examples. Let $\mathcal{T}$ be a triangulated category. Then $\mathcal{T}$ is additive and comes with an automorphism $\Sigma$. We say that a square is special if and only if its fold is a distinguished triangle in $\mathcal{T}$. When we regard a triangulated category $\mathcal{T}$ as a category with squares (see above), then we shall denote it by $\mathcal{T}^{d}$.

Let $\mathcal{A}$ be an Abelian category. Let $G r^{b} \mathcal{A}$ be the category of bounded, graded objects in $\mathcal{A}$. We recall that a graded object of $\mathcal{A}$ is a sequence of objects $\left\{A_{i} \in \mathcal{A}\right\}_{i \in \mathbf{Z}}$. The sequence $\left\{A_{i}\right\}$ is bounded if $A_{i}=0$ except for finitely many $i \in \mathbf{Z}$.

Let the functor $\Sigma: G r^{b} \mathcal{A} \longrightarrow G r^{b} \mathcal{A}$ be the shift, i.e., $\Sigma\left\{A_{i}\right\}=\left\{B_{i}\right\}$ with $B_{i}=A_{i+1}$. A square in $G r^{b} \mathcal{A}$ is said to be special if the fold

$$
A \stackrel{(\alpha,-\beta)^{t}}{\longrightarrow} B \oplus C \stackrel{(\gamma, \delta)}{\longrightarrow} D \stackrel{\mu}{\longrightarrow} \Sigma A
$$

gives a long exact sequence

$$
\cdots \longrightarrow D_{i-1} \longrightarrow A_{i} \longrightarrow B_{i} \oplus C_{i} \longrightarrow D_{i} \longrightarrow A_{i+1} \longrightarrow \cdots
$$

in $\mathcal{A}$.

Let $H: D^{b}(\mathcal{A}) \longrightarrow G r^{b} \mathcal{A}$ be the homology functor taking a complex $A \in D^{b}(\mathcal{A})$ to $\left\{H_{i}(A)\right\}$. Then it induces a functor between categories with squares:

$$
H: D^{b}(\mathcal{A})^{d} \longrightarrow G r^{b} \mathcal{A}
$$

Definition. (1) Let $\mathcal{T}$ be a category with squares, and let $m, n \geq 0$. A functor $X: \Delta^{m} \times$ $\Delta^{n} \longrightarrow \mathcal{T}$ is called an augmented diagram if for any $0 \leq i \leq i^{\prime} \leq m$ and $0 \leq j \leq j^{\prime} \leq n$ we are given a special square

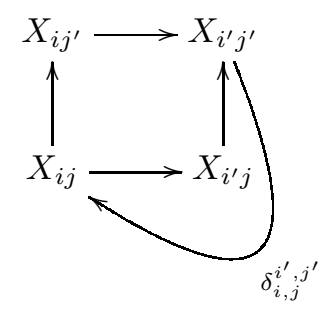

such that $\delta_{i, j}^{i^{\prime}, j^{\prime}}$ is the composition $X_{i^{\prime} j^{\prime}} \longrightarrow X_{m n} \stackrel{\delta_{0,0}^{m, n}}{\longrightarrow} \Sigma X_{00} \longrightarrow \Sigma X_{i j}$. By a morphism $\varphi: X \longrightarrow Y$ between augmented diagrams we mean a natural transformation of functors 
such that the square

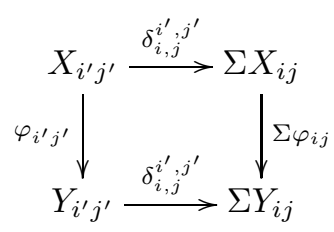

is commutative for any $0 \leq i \leq i^{\prime} \leq m$ and $0 \leq j \leq j^{\prime} \leq n$.

The category of augmented diagrams will be denoted by $Q_{m, n} \mathcal{T}$. This leads to a bisimplicial category $Q \mathcal{T}=\left\{Q_{m, n} \mathcal{T}\right\}_{m, n \geq 0}$ (the face/degeneracy operators are defined by deleting/inserting a row or column).

(2) For a category with squares $\mathcal{T}$, its $K$-theory $K(\mathcal{T})$ is defined to be the space $\Omega|\mathrm{Ob}(Q \mathcal{T})|$.

Let $H: D^{b}(\mathcal{A})^{d} \longrightarrow G r^{b} \mathcal{A}$ be the functor of categories with squares (see above). $H$ induces a map $\chi: Q D^{b}(\mathcal{A})^{d} \longrightarrow Q G r^{b} \mathcal{A}$ of bisimplicial categories and, hence, a map $K(\chi): K\left(D^{b}(\mathcal{A})\right) \longrightarrow K\left(G r^{b} \mathcal{A}\right)$.

Let $\mathcal{E}$ be an exact category, let $m, n \geq 0$, and let $Q_{m, n} \mathcal{E}$ be the following category. Its objects are the functors $X: \Delta^{m} \times \Delta^{n} \longrightarrow \mathcal{E}$ such that for any $0 \leq i \leq i^{\prime} \leq m$ and $0 \leq j \leq j^{\prime} \leq n$ we are given a bi-Cartesian square

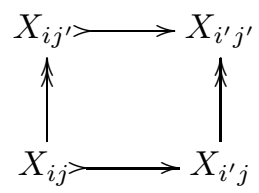

in which the vertical arrows are epimorphisms and the horizontal arrows are monomorphisms. The morphisms are given by natural transformations. The resulting bisimplicial category is denoted by $Q \mathcal{E}$. It is well known that a simplicial model for a delooping of the space $K(\mathcal{E})$ is given by the realization of the bisimplicial set $\mathrm{Ob} Q \mathcal{E}$.

Let $\mathcal{A}$ be an Abelian category, and let $i: \mathcal{A} \longrightarrow D^{b}(\mathcal{A})$ denote the natural functor sending an object $A \in \mathcal{A}$ to the complex concentrated at the zeroth degree. Then it induces a functor (see also some discussion below) of bisimplicial categories $\iota: Q \mathcal{A} \longrightarrow$ $Q D^{b}(\mathcal{A})^{d}$. Note that the differentials $\delta_{i, j}^{i^{\prime}, j^{\prime}}$ in $Q D^{b}(\mathcal{A})^{d}$ are constructed canonically and are unique for every diagram coming from $Q \mathcal{A}$ (see [23]).

Theorem 7.4 (Neeman [22]). Let $\mathcal{A}$ be a small Abelian category. Then the composition

$$
\mathrm{Ob} Q \mathcal{A} \stackrel{\iota}{\longrightarrow} \mathrm{Ob} Q D^{b}(\mathcal{A})^{d} \stackrel{\chi}{\longrightarrow} \mathrm{Ob}_{Q G r^{b}}(\mathcal{A})
$$

is a homotopy equivalence.

As usual, given a category $\mathcal{C}$, we denote by $i \mathcal{C}$ the maximal groupoid in $\mathcal{C}$ and by $i . \mathcal{C}$ the nerve in the $i$-direction.

Corollary 7.5. Let $\mathcal{A}$ be a small Abelian category. Then the composition of maps of trisimplicial objects

is a homotopy equivalence.

$$
i . Q \mathcal{A} \stackrel{\iota}{\longrightarrow} i . Q D^{b}(\mathcal{A})^{d} \stackrel{\chi}{\longrightarrow} i . Q G r^{b}(\mathcal{A})
$$

Proof. For $k \geq 0$, the category $i_{k} \mathcal{A}$ of strings of isomorphisms $A_{0} \stackrel{\sim}{\longrightarrow} \cdots \stackrel{\sim}{\longrightarrow} A_{k}$ is Abelian, and the composition

$$
i_{k} Q \mathcal{A}=Q\left[i_{k} \mathcal{A}\right] \stackrel{\iota}{\longrightarrow} i_{k} Q D^{b}(\mathcal{A})^{d} \stackrel{\chi}{\longrightarrow} i_{k} Q G r^{b}(\mathcal{A})=Q G r^{b}\left[i_{k} \mathcal{A}\right]
$$

is a homotopy equivalence of bisimplicial objects by Theorem 7.4. By Lemma 4.1, so is the map described in the corollary. 
Proof of Theorem 7.1. 1) First, we prove the statement for an Abelian category $\mathcal{A}$. For Quillen's $K$-theory $K(\mathcal{A})$ we use the following simplicial model. It is the loop space of the realization of $i . Q \mathcal{A}$ (see [9]). In its turn, the model for $K\left(\mathbf{D}^{b}(\mathcal{A})\right)$ is given by the bisimplicial maximal groupoid $i Q \mathbf{D}^{b}(\mathcal{A})$ (see $\S 3$ ).

By Corollary 7.5, it suffices to show that the map $i . Q \mathcal{A} \stackrel{\iota}{\longrightarrow} i . Q D^{b}(\mathcal{A})^{d}$ factors through $i . Q \mathbf{D}^{b}(\mathcal{A})$. Recall that $Q_{m, n} \mathbf{D}^{b}(\mathcal{A}), m, n \geq 0$, consists of the objects $X \in \mathbf{D}^{b}(\mathcal{A})_{\Delta^{m} \times \Delta^{n}}$ such that for any $0 \leq i \leq i^{\prime} \leq m$ and $0 \leq j \leq j^{\prime} \leq n$ the square

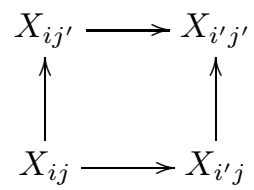

is bi-Cartesian in $\mathbf{D}^{b}(\mathcal{A})_{\square}$ (= co-Cartesian in triangulated dérivateurs, see [7]). It follows that

$$
\operatorname{cone}\left(X_{i j} \longrightarrow X_{i j^{\prime}} \oplus X_{i^{\prime} j}\right) \longrightarrow \operatorname{cone}\left(0 \longrightarrow X_{i^{\prime} j^{\prime}}\right) \simeq X_{i^{\prime} j^{\prime}}
$$

is a quasiisomorphism in $C^{b}(\mathcal{A})$, hence an isomorphism in $D^{b}(\mathcal{A})$ (here we use properties of triangulated dérivateurs and the triangulated structure information encoded by $\mathbf{D}^{b}(\mathcal{A})$, see $\left.[16,5,6]\right)$. Now we compose the inverse of this isomorphism with the natural projection:

$$
\operatorname{cone}\left(X_{i j} \longrightarrow X_{i j^{\prime}} \oplus X_{i^{\prime} j}\right) \longrightarrow \operatorname{cone}\left(X_{i j} \longrightarrow 0\right) \simeq \Sigma X_{i j}
$$

and we have a map $\delta_{i, j}^{i^{\prime}, j^{\prime}}: X_{i^{\prime} j^{\prime}} \longrightarrow \Sigma X_{i j}$. This produces a special square in $D^{b}(\mathcal{A})^{d}$.

Clearly, this construction is natural. Let $f: X \longrightarrow Y$, where $X, Y \in Q_{m, n} \mathbf{D}^{b}(\mathcal{A})$, be an isomorphism. It is represented by a diagram $X \longleftarrow Z \longrightarrow Y$, where $Z \in Q_{m, n} \mathbf{D}^{b}(\mathcal{A})$ and the arrows are quasisomorphisms. We have the following commutative diagram in $C^{b}(\mathcal{A})$ for any $0 \leq i \leq i^{\prime} \leq m$ and $0 \leq j \leq j^{\prime} \leq n$ :

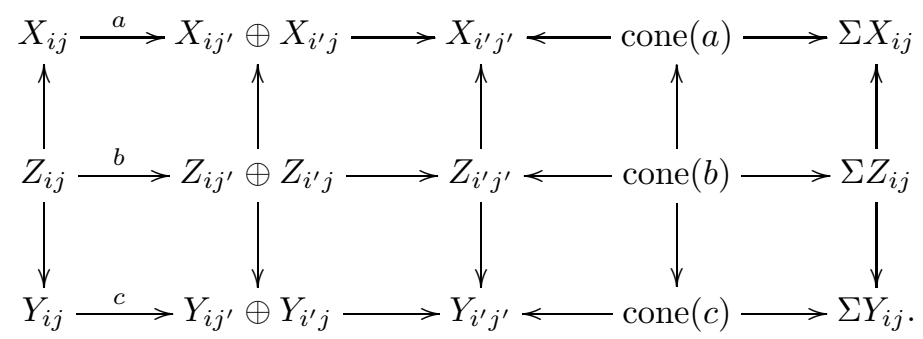

This yields an isomorphism of triangles in $D^{b}(\mathcal{A})$

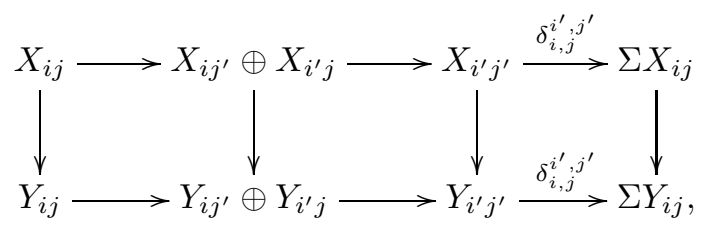

and hence, an isomorphism of special squares in $D^{b}(\mathcal{A})^{d}$.

Now, let $X \in Q_{m, n} \mathbf{D}^{b}(\mathcal{A})$, and suppose that $0 \leq i^{\prime} \leq m$ and $0 \leq j^{\prime} \leq n$. There is a commutative diagram in $C^{b}(\mathcal{A})$,

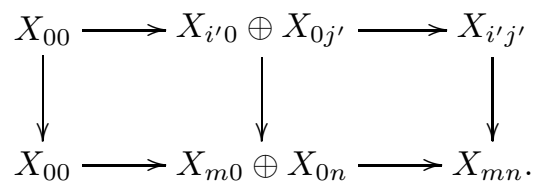


Consequently, in $D^{b}(\mathcal{A})$ we obtain a commutative square

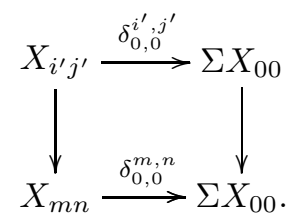

For any $0 \leq i \leq i^{\prime}$ and $0 \leq j \leq j^{\prime}$, in $C^{b}(\mathcal{A})$ there is a commutative diagram

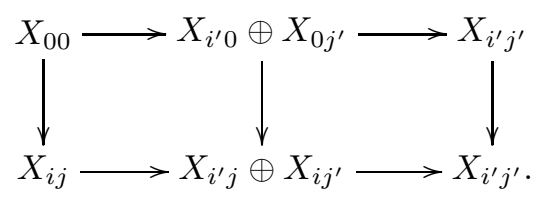

Consequently, in $D^{b}(\mathcal{A})$ we obtain a commutative square

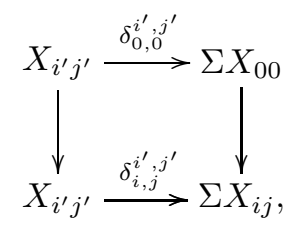

and the "natural" map $\delta_{i, j}^{i^{\prime}, j^{\prime}}: X_{i^{\prime} j^{\prime}} \longrightarrow \Sigma X_{i j}$ is obtained from $\delta_{0,0}^{m, n}: X_{m n} \longrightarrow \Sigma X_{00}$ simply as the composition

$$
X_{i^{\prime} j^{\prime}} \longrightarrow X_{m n} \stackrel{\delta_{0,0}^{m, n}}{\longrightarrow} \Sigma X_{00} \longrightarrow \Sigma X_{i j} .
$$

It follows that the functors dia : $\mathbf{D}^{b}(\mathcal{A})_{\Delta^{m} \times \Delta^{n}} \longrightarrow \operatorname{Hom}\left(\Delta^{m} \times \Delta^{n}, D^{b}(\mathcal{A})\right), m, n \geq 0$, induce a map of bisimplicial groupoids

$$
\theta: i Q \mathbf{D}^{b}(\mathcal{A}) \longrightarrow i Q D^{b}(\mathcal{A})^{d} .
$$

Obviously, the map $i . Q \mathcal{A} \stackrel{\iota}{\longrightarrow} i . Q D^{b}(\mathcal{A})^{d}$ factors as

$$
i . Q \mathcal{A} \stackrel{\rho}{\longrightarrow} i . Q \mathbf{D}^{b}(\mathcal{A}) \stackrel{\theta}{\longrightarrow} i . Q D^{b}(\mathcal{A})^{d} .
$$

This implies the claim.

(2) Suppose now that an exact category $\mathcal{E} \subseteq \mathcal{A}$ satisfies the assumptions of the theorem. Consider the commutative diagram

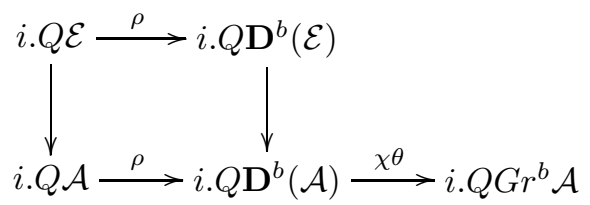

in which the vertical arrows are induced by the inclusion $\mathcal{E} \longrightarrow \mathcal{A}$. The left vertical arrow is a homotopy equivalence by the resolution theorem [28]. Obviously, the fact that the map $\chi \theta \rho$ is a homotopy equivalence (by (1)) completes the proof.

Based on Vaknin's computations in [29], Neeman showed in [23] that there is an exact category $\mathcal{E}$ such that the homomorphism $K_{1}(\iota): K_{1}(\mathcal{E}) \longrightarrow K_{1}\left(D^{b}(\mathcal{E})\right)$ is not a monomorphism (while it is a split monomorphism for Abelian categories [22, 23]). A simplest example is provided by the category $\mathcal{E}$ of free modules of finite rank over the ring of dual numbers $k[\varepsilon] / \varepsilon^{2}$. Such exact categories could give us counterexamples to the first Maltsiniotis conjecture if we showed in a similar way that the map $K_{1}(\rho)$ : $K_{1}(\mathcal{E}) \longrightarrow K_{1}\left(\mathbf{D}^{b}(\mathcal{E})\right)$ is not a monomorphism. 


\section{REFERENCES}

[1] A. Grothendieck, Pursuing stacks, Manuscript, 1983.

[2] L Les dérivateurs, Manuscript, 1983-1990; www.institut.math.jussieu.fr/ maltsin/groth/ Derivateurs.html

[3] A. Heller, Homotopy theories, Mem. Amer. Math. Soc. 71 (1988), no. 383, 78 pp. MR0920963 (89b:55013)

[4] B. Keller, Derived categories and universal problems, Comm. Algebra 19 (1991), no. 3, 699-747. MR1102982 (92b:18010)

[5] J. Franke, Uniqueness theorems for certain triangulated categories with an Adams spectral sequence, K-Theory Preprint Archives, no. 139, 1996.

[6] G. Maltsiniotis, Structure triangulée sur les catégories des coefficients d'un dérivateur triangulé, Exposés au groupe de travail "Algèbre et topologie homotopiques", 2001.

[7] _ La K-théorie d'un dérivateur triangulé, Preprint, 2002; www.institut.math.jussieu.fr/ maltsin.

[8] G. Segal, Categories and cohomology theories, Topology 13 (1974), 293-312. MR0353298 (50:5782)

[9] F. Waldhausen, Algebraic K-theory of spaces, Algebraic and Geometric Topology (New Brunswick, NJ, 1983), Lecture Notes in Math., vol. 1126, Springer-Verlag, Berlin, 1985, pp. 318-419. MR0802796 (86m:18011)

[10] G. Garkusha, Systems of diagram categories and K-theory. II, Math. Z. 249 (2005), no. 3, $641-682$. MR2121745 (2006b:19006)

[11] D.-C. Cisinski and A. Neeman, Additivity for derivator K-theory, Preprint, 2005; www.math. univ-paris13.fr $/{ }^{\sim}$ cisinski.

[12] S. MacLane, Categories for the working mathematician, Grad. Texts in Math., vol. 5, SpringerVerlag, New York, 1998. MR1712872 (2001j:18001)

[13] M. Hovey, Model categories, Math. Surveys Monogr., vol. 63, Amer. Math. Soc., Providence, RI, 1999. MR1650134 (99h:55031)

[14] D.-C. Cisinski, Images directes cohomologiques dans les catégories de modèles, Ann. Math. Blaise Pascal 10 (2003), no. 2, 195-244. MR2031269 (2004k:18009)

[15] K. S. Brown, Abstract homotopy theory and generalized sheaf cohomology, Trans. Amer. Math. Soc. 186 (1974), 419-458. MR0341469 (49:6220)

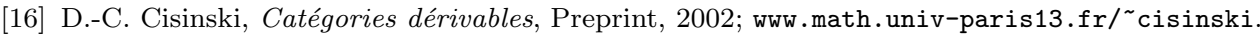

[17] A. K. Bousfield and E. M. Friedlander, Homotopy theory of $\Gamma$-spaces, spectra, and bisimplicial sets, Geometric Applications of Homotopy Theory (Proc. Conf., Evanston, IL, 1977), II, Lecture Notes in Math., vol. 658, Springer-Verlag, Berlin, 1978, pp. 80-130. MR0513569 (80e:55021)

[18] B. Keller, Le dérivateur triangulé associé à une catégorie exacte, Preprint, 2002.

[19] R. W. Thomason and T. Trobaugh, Higher algebraic K-theory of schemes and of derived categories, The Grothendieck Festschrift, Vol. III, Progr. Math., vol. 88, Birkhäuser Boston, Boston, MA, 1990, pp. 247-435. MR1106918 (92f:19001)

[20] D.-C. Cisinski, An e-interchange, January 2004.

[21] F. Waldhausen, Algebraic K-theory of generalized free products, Ann. of Math. (2) 108 (1978), 135-204. MR0498807 (58:16845a)

[22] A. Neeman, K-theory for triangulated categories $3 \frac{1}{2}$, A, B, K-Theory 20 (2000), 97-174; $243-298$. MR1798824 (2002b:18014); MR1798828 (2002b:18015)

[23] The K-theory of triangulated categories, Handbook of $K$-Theory. Vols. 1, 2, Springer-Verlag, Berlin, 2005, pp. 1011-1080. MR2181838 (2006g:19004)

[24] B. Toën, Homotopical and higher categorical structures in algebraic geometry, Habilitation thesis, Preprint math.AG/0312262.

[25] B. Toën and G. Vezzosi, A remark on K-theory and S-categories, Topology 43 (2004), no. 4, 765791. MR2061207 (2005e:19001)

[26] B. Toën, Comparing S-categories and "dérivateurs de Grothendieck", Preprint, 2003; math.unice. $\mathrm{fr} /{ }^{\sim}$ toen.

[27] M. Schlichting, A note on K-theory and triangulated categories, Invent. Math. 150 (2002), 111-116. MR1930883 (2003h:18015) 
[28] D. Quillen, Higher algebraic K-theory. I, Algebraic K-Theory, I: Higher K-Theories (Proc. Conf., Battelle Memorial Inst., Seattle, Wash., 1972), Lecture Notes in Math., vol. 341, Springer-Verlag, Berlin, 1973, pp. 85-147. MR0338129 (49:2895)

[29] A. Vaknin, Determinants in triangulated categories, K-Theory 24 (2001), 57-68. MR1865601 (2002i:19002)

Department of Mathematics, University of Wales Swansea, Singleton Park, SA2 8PP Swansea, United Kingdom

E-mail address: garkusha@imi.ras.ru

Received 8/MAR/2006 